\title{
PHILOSOPHICAL TRANSACTIONS OF THE ROYAL SOCIETY B
}

BIOLOGICAL SCIENCES

\section{Brain stimulation to left prefrontal cortex modulates attentional orienting to gaze cues}

\begin{tabular}{|r|l|}
\hline Journal: & Philosophical Transactions B \\
\hline Manuscript ID & RSTB-2018-0430.R2 \\
\hline Article Type: & Research \\
\hline $\begin{array}{r}\text { Author: } \\
\text { Date Submitted by the }\end{array}$ & n/a \\
\hline $\begin{array}{r}\text { Complete List of Authors: } \\
\begin{array}{r}\text { Issue Code (this should have } \\
\text { please contact the Editorial } \\
\text { Office if it is not present): }\end{array}\end{array}$ & $\begin{array}{l}\text { Abubshait, Abdulaziz; George Mason University, Psychology } \\
\text { Azarian, Bobby; George Mason University, Psychology } \\
\text { Blumberg, Eric Joshua; George Mason University, Psychology }\end{array}$ \\
\hline Subject: & Cognition < BIOLOGY, Neuroscience < BIOLOGY, Behaviour < BIOLOGY \\
\hline Keywords: & $\begin{array}{l}\text { human-robot interaction, mind perception, brain stimulation, social } \\
\text { attention }\end{array}$ \\
\hline
\end{tabular}

\section{SCHOLARONE ${ }^{\text {TH }}$ \\ Manuscripts}




\title{
Brain stimulation to left prefrontal cortex modulates attentional orienting to gaze cues
}

\author{
Eva Wiese*, Abdulaziz Abubshait*, Bobby Azarian \& Eric J. Blumberg \\ George Mason University \\ *shared first authorship
}

\section{Corresponding author:}

Eva Wiese

Department of Psychology

Social and Cognitive Interactions Lab

George Mason University

ewiese@gmu.edu 


\begin{abstract}
MEDIA SUMMARY
Social robots are increasingly becoming a part of everyday life, and researchers are confronted with the challenge of how to make them sufficiently social for humans to accept them as social companions. Previous research has shown that the effect of nonverbal signals like changes in gaze direction on social-cognitive processes in human-robot interaction depends on the degree to which the robot is perceived as "having a mind". In using brain stimulation, the paper shows that this modulatory effect of mind perception on social-cognitive processes in human-robot interaction likely originates from left prefrontal brain areas.
\end{abstract}




\begin{abstract}
In social interactions, we rely on nonverbal cues like gaze direction to understand the behavior of others. How we react to these cues is determined by the degree to which we believe that they originate from an entity with a mind capable of having internal states and showing intentional behavior, a process called mind perception. While prior work has established a set of neural regions linked to mind perception, research has just begun to examine how mind perception affects social-cognitive mechanisms like gaze processing on a neuronal level. In the current experiment, participants performed a social attention task (i.e., attentional orienting to gaze cues) with either a human or a robot agent (i.e., manipulation of mind perception) while transcranial direct current stimulation (tDCS) was applied to prefrontal and temporo-parietal brain areas. The results show that temporo-parietal stimulation did not modulate mechanisms of social attention, neither in response to the human nor the robot agent, whereas prefrontal stimulation enhanced attentional orienting in response to human gaze cues and attenuated attentional orienting in response to robot gaze cues. The findings suggest that mind perception modulates low-level mechanisms of social cognition via prefrontal structures, and that a certain degree of mind perception is essential in order for prefrontal stimulation to affect mechanisms of social attention.
\end{abstract}




\section{INTRODUCTION}

People are seeing a day-to-day increase in the number of robot agents in their lives that could assist them in various domains [1]. Although evidence of positive outcomes of humanrobot interactions exist [2-4], designing social robots that elicit natural human responses can be challenging due to people's negative perceptions about having social robots be a part of their everyday life $[5,6]$. To remedy this and design robots that are able to elicit social responses, we must understand how the human brain processes social information in interactions with others and whether nonhuman agents are able to activate these networks to a similar extent as human interaction partners (and if so, under which conditions). Following this approach, we can identify physical and behavioral agent features that reliably activate brain areas involved in social-cognitive processes and investigate whether robots that elicit activation in these networks lead to more acceptance and trust, as well as improved performance in human-robot interaction [58].

Meaningful social interactions require the ability to infer internal states of others, such as intentions (i.e., mentalizing) and emotions (i.e., empathizing) [7], and to use this information to predict future behavior [8]. For that purpose, the human brain is equipped with neural networks specialized in processing information relevant to social interactions (i.e., social brain; [8-10]) that involve posterior areas like the temporo-parietal junction (TPJ), the superior temporal sulcus (STS) and the fusiform gyrus (FG), as well as anterior areas like the anterior cingulate cortex (ACC) and the ventromedial and dorsolateral prefrontal cortex (vmPFC, dIPFC) [8,11-16]. TPJ is involved in inferring higher-order action goals [17-25] and mental and spatial perspective taking $[22,24,26]$, while STS and FG are involved in processing biological motion and 
face identity, respectively $[8,15]$. PFC is involved in making inferences about enduring dispositions such as preferences or beliefs $[14,23,27,28]$, and activation in medial PFC is positively correlated with the amount of background knowledge we have about others $[18,29]$. $A C C$ is activated in social interactions requiring mentalizing in real-time $[25,30]$, and is more strongly activated when interacting with human versus machine agents (i.e., computers; [31]). Critically for human-robot interaction, most current social robot platforms underactivate the social brain network [31,41,44], which negatively impacts social (e.g., joint attention), emotional (e.g., empathy) and cognitive (e.g., trust) processes that would be essential in order for humans to socially interact with robot agents. Fortunately, for social roboticists, social brain activation depends on the degree to which an interaction partner is perceived as "having a mind" (i.e., mind perception [32]), with the general capability of making changes in the environment (i.e., agency; [33]), and experiencing internal states, such as emotions and intentions (i.e., experience), and as such can presumably be triggered by design. Whereas mind is easily perceived in other human agents [34], the degree to which nonhuman entities like robots trigger mind perception depends on whether their physical and behavioral characteristics are perceived as sufficiently human-like [35-38]. Mind perception is in fact a highly effortless process that activates social brain networks in a bottom-up or reflexive fashion [133-136], triggered by human-like facial features and relations (i.e., spatial arrangement of eye-nose-mouth configurations) $[131,132,134,135,137]$, as well as biological motion and/or predictable behavior [49].

Due to its reflexive nature, mind perception allows observers to differentiate intentional from non-intentional agents within a few hundred milliseconds $[135,136]$, and even just 
passively viewing stimuli that trigger mind perception is sufficient in order to induce activation in a wide range of social brain networks [126], which varies parametrically as a function of the agent's physical human-likeness (i.e., increases as the agent's face or body becomes more human-like in appearance) $[57,84]$. Brain areas related to variations in mind perception involve anterior social brain areas, such as the left ACC $[12,25,43]$, as well as posterior areas, such as the left TPJ [90]. Left ACC is activated when others are treated as intentional agents [43, 141], and responds more strongly during social decision making tasks that involve intentional versus non-intentional agents $[43,141]$; activation within left TPJ is associated with attributing humanlikeness and intentionality to non-human agents [138-140], and gray matter volume in left TPJ corresponds to individual differences in anthropomorphizing non-human entities, in particular animals [90]. The degree to which agents trigger mind perception not only modulates activation in social brain areas, it also determines how we feel about them [39-42], behave towards them $[31,43-46]$ and interact with them [47-50], and as such has the potential to impact acceptance, trust and performance in human-robot interactions. Mind perception affects higher-order social-cognitive processes like prosociality, morality and economic decision-making [46,49], and also modulates low-level social-cognitive processes, such as face perception [51] and social attention [52-55]. The effect of mind perception on social cognition is so profound that the mere belief that observed behavior may reflect the actions of an agent "with a mind" makes people interpret pre-programmed behaviors as intentional and motivates them to attune their actions accordingly [44,54-56].

Although the neural link between mind perception and social brain activation [27,57], as well as the behavioral link between mind perception and social-cognitive processes are 
relatively well understood [58], research has just begun to examine how mind perception modulates social cognition on a neural level (i.e., activation of which brain areas modulates social cognition as a function of mind perception) [44]. To address this important issue, we use transcranial direct current stimulation (tDCS) to investigate the link between activation of social brain areas implicated in mind perception and low-level social cognitive processes, such as social attention (i.e., the degree to which changes in gaze direction are followed) [67]. TDCS is a non-invasive electrical stimulation technique that can be applied without disrupting the participant during task execution, and has been proven to be effective in modulating a wide range of cognitive processes in previous studies (e.g., memory, attention, decision-making, perception; [59-62]). Social attention, or the tendency to follow changes in others' gaze direction, was chosen for the present study, as it is a basic, yet essential, social-cognitive mechanism that allows for initiating and coordinating communication [63-65], establishing joint attention between two interaction partners and an object of interest in the environment [66], and an important precursor for developing a theory of mind [66].

Social attention can be examined using a gaze-cueing paradigm, where a face-like stimulus is presented centrally on a screen that first looks straight and then changes gaze direction to the left or right side of the screen. This so-called gaze cue is followed by the presentation of a target stimulus (e.g., F or T) that either appears at the cued location (i.e., valid trial) or an uncued (i.e., invalid trial) location and triggers shifts of the observer's attention to the gazed-at location. As a result, reaction times on valid trials are usually shorter than reaction times on invalid trials, with the difference in reaction times between invalid and valid conditions constituting the gaze-cueing effect [67]. Although it is widely accepted that social 
attention has a strong bottom-up component (i.e., attention is shifted reflexively to the cued location; [66]), there is accumulating evidence that it can be top-down controlled by higherorder social-cognitive processes when context information is available that increases the social relevance of observed gaze signals (e.g., gazer is similar or known to the observer; [54,68-77]). With particular relevance to the current study, manipulating the degree to which a gazer is perceived as an intentional being "with a mind" has been shown to modulate social attention, such that gaze-cueing effects are larger in response to human (i.e. intentional) versus machine (i.e., pre-programmed) gazers $[54,55,78,79]$. That this top-down modulation is specifically related to mind perception is supported by experiments showing that although individuals on the autism spectrum reflexively attend to gaze signals, they do not show the reported enhancement of gaze-cueing effects for human agents but an enhancement in attentional orienting in response to robot gaze cues (potentially due to difficulties with inferring internal states underlying human gaze behavior but an increased interest in machine behavior) [79]. While modulatory effects of mind perception on social attention have been examined behaviorally $[46,48]$, how mind perception modulates lower-level social-cognitive mechanisms like gaze cueing at the neuronal level still remains an open question. In particular, it is unclear whether anterior or posterior parts of the social brain network are more strongly involved in modulating social cognition via mind perception. On the one hand, Oezdem et al. showed that believing that an agent's eye movements are human-controlled (i.e., intentional) as opposed to pre-programmed (i.e., non-intentional) modulated activation in bilateral TPJ (but not prefrontal networks), and enhanced attentional orienting to gaze cues [44]. On the other hand, Wiese et al. showed that although variations in social attention in response to human versus robot 
gazers correlated with activation in anterior (i.e., ventromedial PFC) and posterior social brain areas (i.e., TPJ), only activation in bilateral vmPFC correlated with both variations in mind perception and social attention, suggesting that vmPFC might be involved in the top-down control of social attention via mind perception [57]. Taken together, these findings show that although there is convincing evidence that social attention can be modulated via mind perception, the exact source of this modulatory effect still needs to be identified.

To address inconsistencies of previous studies and to examine whether anterior and/or posterior areas of the social brain network implicated in mind perception are causally involved in modulating social attention, in the current study we compare gaze-cueing effects induced by agents "with a mind" (i.e., human) to agents "without a mind" (i.e., robot), with and without tDCS stimulation to left prefrontal and left temporo-parietal areas. This manipulation was chosen for two reasons: First, previous studies showed that sophisticated minds are attributed to agents with human-like appearance but not to agents with a robot-like appearance (i.e., mind ratings increase as a function of physical human-likeness $[33,81-83])$, which allows us to experimentally manipulate mind perception via physical human-likeness. Second, since mind perception increases activation in social brain networks [84-87] and has been shown to modulate social-cognitive processes like gaze cueing on a behavioral level $[55,88]$, using tDCS in the context of a social attention paradigm seems suitable to investigate the outlined research goal. Left prefrontal and left temporo-parietal areas were chosen as sites for tDCS stimulation for the following reasons: First, both areas have been implicated in mind perception in previous studies $[27,44,57,43,141]$, and activation in both areas has been shown to correlate with variations in social attention (i.e., gaze cueing) $[44,57]$. Second, both areas are located distant 
enough from each other to minimize the risk of accidental stimulation of the respective other site during stimulation (i.e., prefrontal vs. temporo-parietal). Third, previous studies suggest that active stimulation is superior to sham stimulation as a control, as it controls for side effects of stimulation that are not directly associated to brain functionality like stimulation sensations, and allows for drawing specific inferences regarding the origin of the modulation in terms of location, as permitting by the low spatial resolution of tDCS targeting [92,93].

\section{METHODS AND MATERIALS}

\section{Participants}

Eighty-two undergraduate students from George Mason University (57 females, $M=19.84, \mathrm{SD}=$ 2.35 , range: $18-29$ years) participated in the current study for course credit. All participants were right-handed, had normal or corrected-to-normal vision, had no known neurological or psychological deficits, were not taking any medications known to affect the central nervous system at the time of the experiment, and had no history of migraines, seizures, or head injuries. All participants provided written consent to participation and were debriefed at the end of the study. Collection and handling of participant data were in accordance with the IRB guidelines (obtained prior to data collection). Data of participants whose accuracy rate in the gaze cueing task during baseline was below $85 \%$ ( 6 participants), who did not follow the instructions properly ( 2 participants), or did not complete the study (2 participants) were excluded from data analysis ( $13 \%$ of participants in total). The remaining 72 participants were quasi-randomly assigned to the experimental condition, that is: active stimulation of the left prefrontal cortex (PFA; $\mathrm{n}=36,23$ females), or the control condition, that is: stimulation of the 
left temporo-parietal cortex (TPA; $n=36,27$ females). To determine the sample size needed for the study, an a priori power analysis was conducted in $\mathrm{G}^{*}$ power for a mixed-effects repeated measures design (i.e., one between factor and one within factor). Since mixed-effects repeated measures designs in $\mathrm{G}^{*}$ power can only handle two-factorial designs, the sample size was determined for an ANOVA with a 2-level between factor and a 4-level within factor (i.e., the two within variables were combined). The analysis was based on an alpha of $(\alpha=.05)$, the power set to $(1-\beta=.95)$, and the assumption of a small-to-medium effect size (Cohen's $d=$ .17). The analysis showed that an approximate sample size of 76 participants would be sufficient for both experimental groups. Since the current version of G*power cannot test for a 3-way factorial design but only a 2-way factorial design, another power analysis was conducted using $\mathrm{R}$ for the general linear model, which is a more generic test. The analysis was done for a $2 \times 2 \times 2$ factorial model that contains three main effects and their interactions (i.e., three 2-way interactions and one 3-way interaction) using the same alpha $(\alpha=.05)$, beta $(1-\beta=.95)$, and effect size (Cohen's $d=.17$ ) as the previous analysis. The analysis for the general linear model revealed that the study needed a total of 83 participants.

\author{
Apparatus \\ Stimuli were presented on a Dell $1703 \mathrm{FP}$ monitor with the refresh rate set at $85 \mathrm{~Hz}$. RT \\ measures were based on standard keyboard responses. Participants were seated approximately \\ $57 \mathrm{~cm}$ from the monitor, and the experimenter ensured that participants were centered with \\ respect to the monitor. The experiment was controlled by Experiment Builder (SR Research Ltd., \\ Ontario, Canada).
}




\section{TDCS stimulation}

An ActivaDose II, ActivaTek system was used to administer the $2 \mathrm{~mA}$ stimulation. Although there is not a universal and ideal level of current stimulation for any one montage, we selected $2 \mathrm{~mA}$ because it is a commonly used level that is within human safety limits [94]. Electrode placement followed the 10-5 EEG system [95], and tDCS was delivered via two $5 \times 5 \mathrm{~cm}$ saline soaked sponges in rubber housing (resulting in a sponge contact area of $3 \times 3 \mathrm{~cm}$ ). For PFA stimulation, the anode was placed on the scalp over F9 and the cathode was placed over Fz. For TPA stimulation, the anode was placed on the scalp over P5 and the cathode was placed extracephalically on the right $[96,97]$. This montage was chosen to minimize erroneous stimulation of non-targeted cortical areas based on brain modeling (see Brain Modeling; for more details).

\section{Brain Modeling}

Stimulation parameters were modeled using Finite Element Models (FEM) within the HD Explore brain modeling software (Soterix Medical, NY, USA). Brain models were obtained based on conductivities from Datta et al., [98]. The brain models for PFA and TPA stimulation were created for $5 \times 5 \mathrm{~cm}$ sponges (as required by Soterix software). However, since the sponges were reduced to $3 \times 3 \mathrm{~cm}$ due to the usage of sponge housings, we used a 160-channel EEG cap to measure the number of electrodes that were not targeted by the $3 \times 3 \mathrm{~cm}$ sponges in order to correct the initial brain models by reducing the number of electrodes stimulated in the brain modeling software to provide the best approximation of the stimulation intensity at each area. 
PFA stimulation reached multiple prefrontal structures, including the left medial prefrontal cortex (IMPFC), dorsolateral prefrontal cortex (IDLPFC), dorsomedial prefrontal cortex (IDMPFC), ventromedial prefrontal cortex (IVMPFC), and anterior cingulate cortex (IACC): Soterix showed an average peak stimulation intensity of $0.34 \mathrm{~V} / \mathrm{m}$ per $1 \mathrm{~mA}$. For TPA stimulation, structures that received stimulation included the left temporo-parietal junction (ITPJ), left superior temporal sulcus (ISTS), left precuneus (IPRC), and left fusiform face area (IFFA): Soterix showed an average peak stimulation intensity of $0.33 \mathrm{~V} / \mathrm{m}$ per $1 \mathrm{~mA}$ (see Figure 1 for brain models and Supplementary Materials for exact stimulation intensities of each structure).

Although multiple models were evaluated, the electrode montages for PFA and TPA stimulation reported under "tDCS stimulation" demonstrated the greatest peak stimulation intensity over the targeted brain regions, with the least amount of stimulation to non-target brain regions. Specifically, FEM modeling illustrated that using an extracephalic cathodal electrode reduces erroneous stimulation for non-targeted cortical areas in the TPA stimulation condition. It is important to note that although recent evidence suggests that an extracephalic electrode may reduce the magnitude of the stimulation effect when compared to scalp placements [99], this is not the case in the current experiment as FEM models suggest that similar stimulation intensities were achieved for PFA and TPA stimulation $(0.34 \mathrm{~V} / \mathrm{m}$ vs 0.33 $\mathrm{V} / \mathrm{m}$ per $1 \mathrm{~mA})$. 


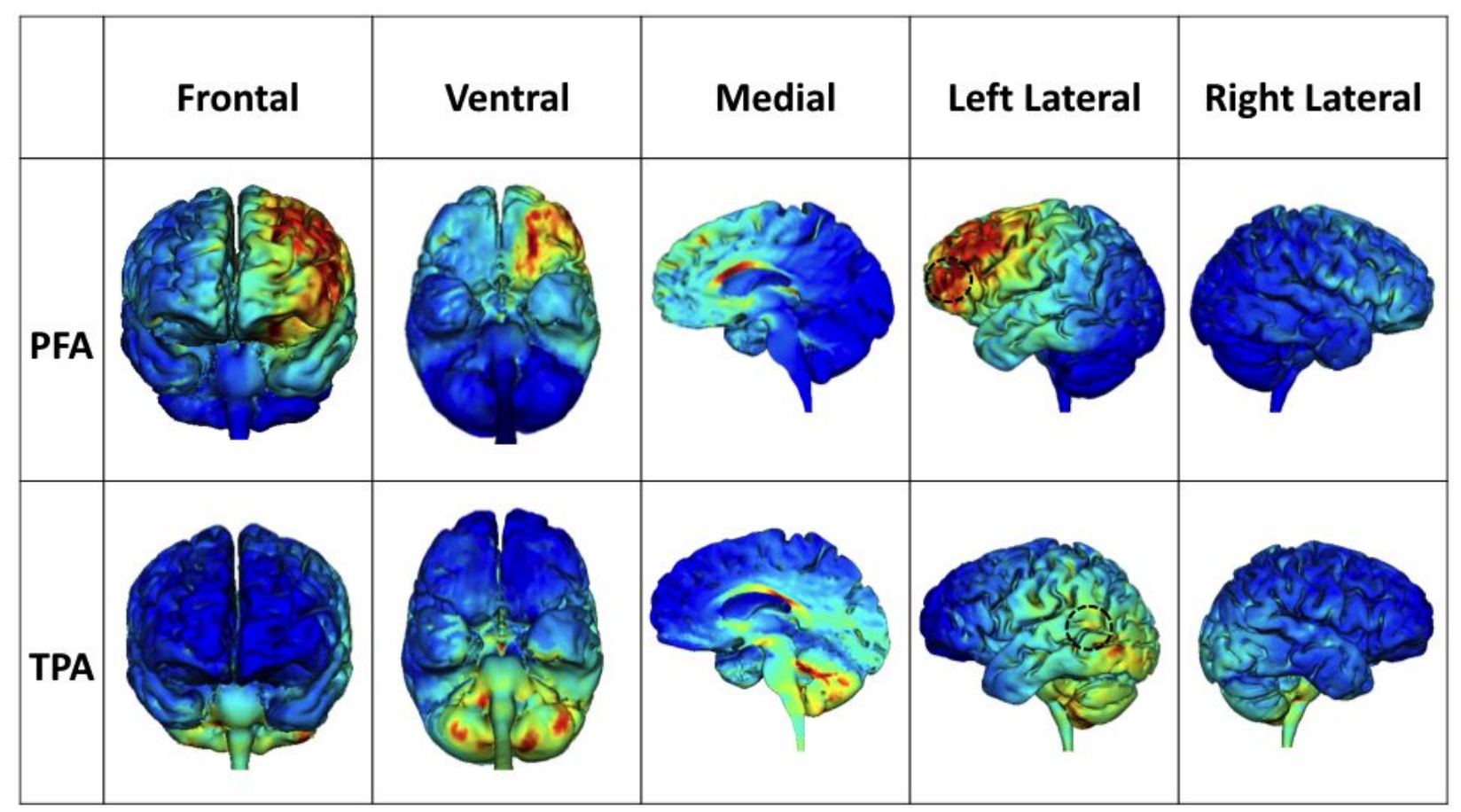

Figure 1. Brain Models illustrate the field intensities of stimulation in $\mathrm{V} / \mathrm{m}$ per $\mathrm{mA}$. Darker red areas illustrate higher stimulation intensities compared to blue areas. The circled regions in the Left Lateral pane show the PFA region and the TPA region, respectively. Please see Figure $\mathbf{1}$ for brain models and Supplementary Materials for exact stimulation intensities of each structure.

\section{Stimuli}

Gaze cueing requires participants to detect, locate or identify targets that are looked-at or looked-away from by a gazer [67], which in the current study is either a human (mind perception high) or a robot (mind perception low). In the human condition, the digitized photo of a female face was used as a gazer, which can be found in the Karolinska Directed Emotional Faces database, while in the robot condition the photo of a humanoid robot was used as a gazer (EDDIE; developed by the Technical University of Munich, Germany). The gazing stimuli were $6.4^{\circ}$ wide and $10.0^{\circ}$ high, depicted on a white background and presented in full frontal orientation with eyes positioned on the central horizontal axis of the screen (Figure 2). For left- 
and rightward gaze, irises and pupils of the human and the robot gazer were shifted with Photoshop and deviated $0.4^{\circ}$ from direct gaze. The target stimulus was a black capital letter ( $\mathrm{F}$ or $\mathrm{T}$; measuring $0.8^{\circ}$ in width and $1.3^{\circ}$ in height), which participants had to discriminate by pressing assigned keys on a regular keyboard. The target letters appeared on the horizontal axis of the screen and were located $6.0^{\circ}$ left or right from the center of the screen.

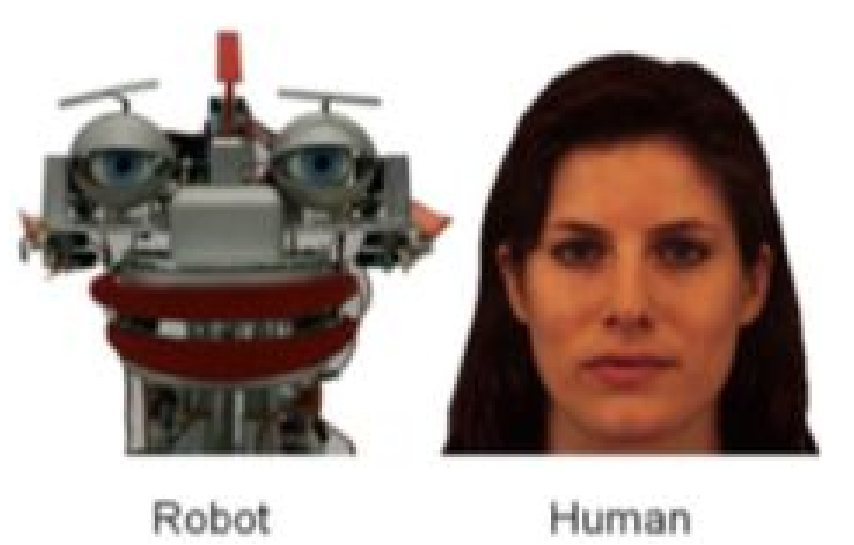

Figure 2. Human and Robot Stimuli: the human agent is represented by a female face taken from the Karolinska Directed Emotional Faces (KDEF) database (F07; written informed consent from Karolinska Institute was received to use the photograph for experimental investigations and illustrations). The robot agent is the robot EDDIE (developed at the Technical University of Munich).

\section{Procedure}

At the beginning of the experiment, participants gave informed consent and completed the Snellen near-sightedness exam to test their eye vision. They then answered a set of questionnaires to assess their perception of robots from the Godspeed measure [100], attitudes towards robots from the Negative Attitude towards Robots questionnaire [101], and autistic traits from the Autism quotient [102]. Upon completion of the questionnaires, participants were instructed how to perform the gaze cueing task, and were informed that the experiment consisted of two parts: a first part where they would perform the gaze cueing task 
without stimulation, and a second part, where they would perform the gaze cueing task under stimulation, with a break for setting up the tDCS equipment in between.

After completing the baseline block, which took 20 minutes, the researcher started setting up the tDCS equipment, which took about 10 minutes. As soon as the current reached its maximum value of $2.0 \mathrm{~mA}$, participants completed a sensation questionnaire to monitor their comfort levels. They were given an unrelated video-game experience questionnaire following the first sensation questionnaire. The unrelated video game questionnaire was administered to ensure that the timing of the stimulation was similar to previous studies that successfully modulated cognitive processes using tDCS $[96,103-106]$. This was an important step as there is no unified standard for the use of tDCS modulation of cognitive tasks [107]. After completing the unrelated videogame questionnaire, participants completed a second sensation questionnaire. Next, subjects started the stimulation gaze-cueing block, which also took 20 minutes. After the stimulation gaze-cueing block, a third sensation questionnaire was administered, the electrodes were removed, the GSM and NARS questionnaire were administered again, and the participants were debriefed. The timing of the experiment can be viewed in Figure 3. 

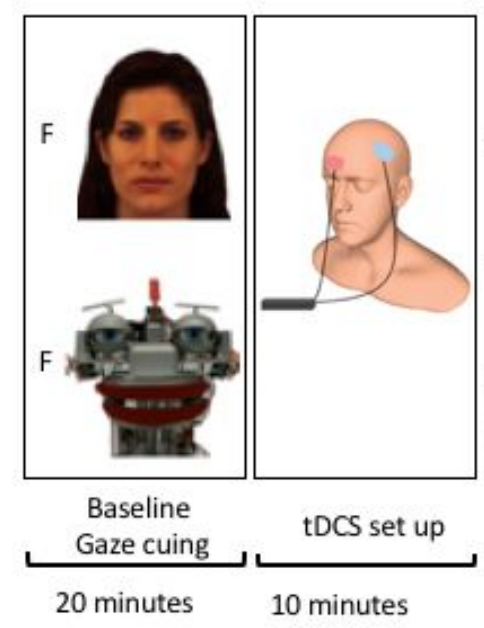

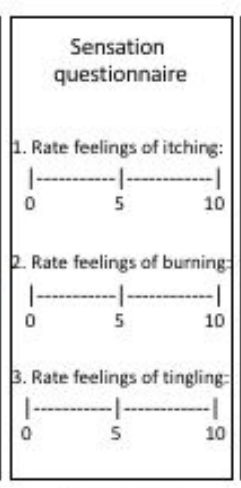

\section{Sensation} questionnaire

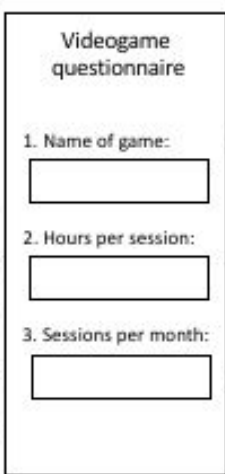

Break questionnaire 10 minutes

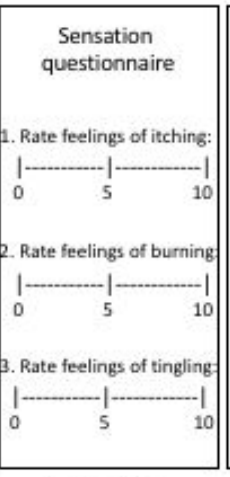

Sensation questionnaire

$\sim 1$ minute

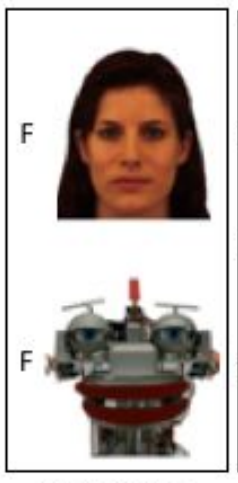

Stimulation Gaze cueing 20 minutes

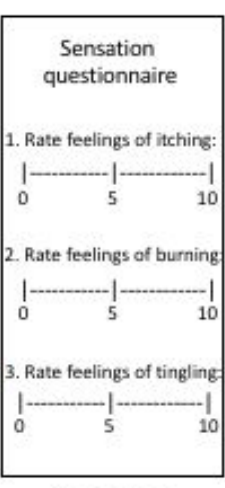

Sensation questionnaire

$\sim 1$ minute

Figure 3. Timing of the Experimental Procedure: The experiment started with participants completing the baseline gaze-cueing task. Next the researcher set up the tDCS machine and participants completed a questionnaire about their sensations. The participants then completed a decoy survey, which asked about their video-game experience. Participants then completed a second sensation questionnaire followed by a gaze-cueing task under stimulation. After the stimulation gaze cueing task was completed, a final sensation questionnaire was administered and the tDCS stimulation was stopped (set-up image was adapted from Dayan et al., 2013).

The sequence of events on a given trial of gaze cueing is illustrated in Figure 4. The beginning of each trial was signaled by a fixation cross at the center of the screen. Between 700-1000 ms later, one of the agents (i.e., human or robot) appeared on the screen looking straight (and with the fixation cross remaining in its position). After a random time interval of 700-1000 ms, the gazer shifted its gaze either left- or rightwards, which constituted the gaze cue. After a stimulus onset asynchrony (SOA; i.e., time interval between gaze cue and target onset) of 400-600 ms, a target letter ( $F$ or T) appeared on the left or the right side of the screen, and participants were asked to respond as fast and accurately as possible to the identity of the 
target [67]. ${ }^{1}$ Gaze cue and target letter remained on the screen until a response was given or after a timeout of 1200 ms was reached, whichever came first. The next trial started after an inter-trial-interval (ITI) of $680 \mathrm{~ms}$.

Participants were instructed to fix their gaze on the fixation cross at the beginning of a trial, and to not make any eye movements during the trial. They were also instructed that after the fixation cross the image of a social agent would appear in the center of the screen, which would first look at them (mutual gaze), and then after some time make an eye movement to look to the left or right side of the screen (averted gaze). Participants were further advised that the change in gaze direction would be followed by the appearance of a target letter ( $F$ or T), which would appear either at the gazed-at location or opposite of the gazed-at location. Participants were asked to indicate as quickly and accurately as possible whether " $\mathrm{F}$ " or " $\mathrm{T}$ " was shown on the screen by pressing the respective response key: For one half of the participants " $\mathrm{F}$ " was assigned to the " $\mathrm{D}$ " key and " $\mathrm{T}$ " to the " $\mathrm{K}$ " key on a regular keyboard, while for the other half of participants stimulus-response mapping was reversed. The original key labels were covered with a sticker to prevent interference effects with the actual letters on the keyboard. All instructions were given in written form.

Gaze direction (left, right), target location (left, right), target identity $(F, T)$ and agent type (human, robot) were selected pseudo-randomly; every combination appeared with equal frequency throughout the experiment. Gaze direction was manipulated orthogonal to target

\footnotetext{
${ }^{1}$ The SOAs were jittered to prevent preparedness effects on the participant's side. Please note that this is in line with previous studies (e.g., [55]) and should not affect attentional orienting, since no qualitative differences in attentional orienting have been reported in this time frame (i.e., both reflexive and voluntary shifts of attention occur in the 400-600 ms SOA range, and not inhibition of return effects have been reported) [66].
} 
location: in half of the trials, the target was validly cued, and in the other half of the trials, the target was invalidly cued. Each experimental session was composed of 220 trials, with a block of 20 practice trials preceding two gaze-cueing blocks of 100 trials of gaze cueing each (i.e., 220 trials for the stimulation baseline and 220 trials for the stimulation block). Participants first completed one block of gaze cueing without stimulation, and were then assigned to either the group that received active stimulation to left PFA or the group that received active stimulation to left TPA. Stimulation was applied for 30 minutes, during which participants completed the second block of gaze cueing.

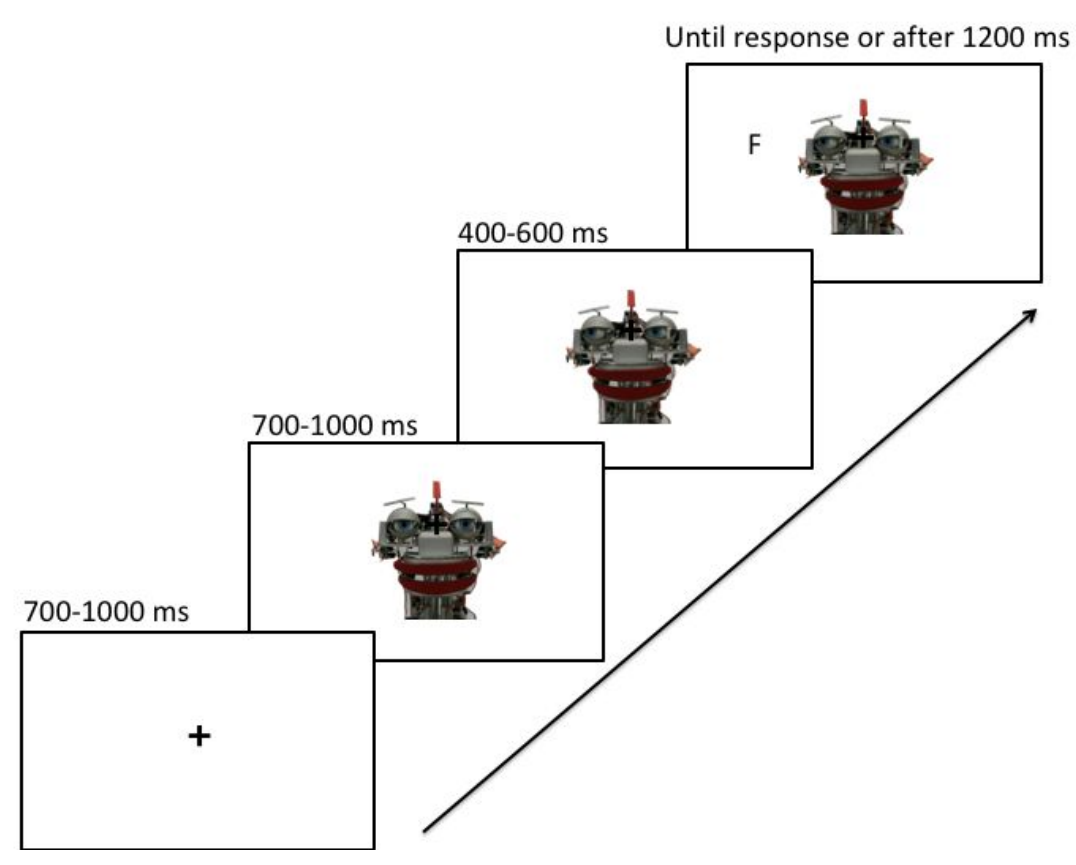

Figure 4. Sequence of events on a trial of gaze cueing: participants first fixated on a fixation cross for 700-1000 ms and were then presented with the gazing agent (human vs. robot) looking straight for 700$1000 \mathrm{~ms}$, followed by a change in gaze direction (either to the left or right side of the screen). After an SOA of 400-600 ms, the target letter (F or T) appeared either where the face was looking or opposite of where the face was looking. The target remained on the screen until a response was given or a timeout of 1200 ms was reached. 


\section{RESULTS \\ Questionnaires}

We used the Godspeed Measure (GSM; [100]) and the Negative Attitude towards Robots Scale (NARS; [101]) to determine whether participants in the two different stimulation conditions differed in their perception of and attitudes towards robots. Both questionnaires were administered at the beginning of the experiment (to capture potential a-priori individual differences), as well as after the completion of the experiment (to assess whether attitudes changed). The short version of the Autism Quotient (AQ-short; [102]) was administered once, at the beginning of the experiment, to measure participants' autistic traits. A 10-point sensation questionnaire was also administered three times to monitor participant's comfort levels during the experiment $[96,97]$. Participants were told that a reporting a "7" would illustrate unbearable sensations. The questionnaire was administered as soon as stimulation started, before the start of the stimulation block, and after completing the stimulation block. Participants in the PFA and the TPA stimulation did not differ in terms of their perception of robots $\left(G S M ; F(1,69)=.09, p=.75 ; \eta^{2}<.001\right)$, attitudes towards robots (NARS; $F(1,69)=.2$, $\left.p=.65 ; \eta^{2}<.01\right)$ or autistic traits (AQ score; $\left.t(69)=-1.66, p=.09\right)$. Comparison of the GSM and NARS pre-post ratings did not reveal differences between the two stimulation conditions (GSM: $F(1,69)=1.19, p=.27, \eta^{2}<.01 ;$ NARS: $\left.F(1,69)=.04, p=.84, \eta^{2}<.001\right) ;$ see Tables S1 and S2 in the Supplementary Materials for the full report of the inference statistics. Two participants indicated sensation levels of above "7", which indicated that they were uncomfortable and did not continue the experiment (see participants section). 


\section{Behavioral Data}

To determine whether active PFA and/or TPA stimulation had a modulatory effect on gaze cueing, we conducted a $2 \times 2 \times 2$ mixed ANOVA on gaze cueing effects (i.e., mean reaction times for invalid - valid trials) with Agent Type (human vs. robot) and Session (baseline vs. stimulation) as within-participants factors, and Brain Site (left PFA vs. TPA) as between-participants factor. The descriptive statistics of this analysis are depicted in Figure $\mathbf{5 .}$

Before examining the results of the statistical models, we tested the normality of the residuals using the Kolmogorov-Smirnov test (and not the more commonly used Shapiro-Wilk test as is it not recommended for larger sample sizes; $[108,109])$. The Kolmogorov-Smirnov test revealed a non-significant effect $(D=.07, p=.42)$, showing that the residuals of our data did not differ significantly from a normal distribution (i.e., the normality of the residuals assumption of parametric tests was not violated).

The ANOVA revealed no main effect of Agent Type $\left(F(1,70)=.2, p=.64, \eta^{2}=<.001\right)$, indicating that across stimulation sites and sessions, there were no significant differences in gaze-cueing effects for the human agent compared to the robot agent (Human: 8.22 ms vs. Robot: $7.20 \mathrm{~ms})$. The main effect of Session $\left(F(1,70)<.001, p=.98, \eta^{2}<.001\right)$ was also not significant, showing that across stimulation sites and agent types gaze cueing effects did not differ between baseline and stimulation (Baseline: 7.69 ms vs. Stimulation: 7.74 ms). The main effect of Brain Site $\left(F(1,70)<.001, p=.98, \eta^{2}<.001\right)$ was also not significant, showing that across agent types and sessions, no differences in gaze cueing effects were found between the two stimulation sites (PFA: 7.69 ms vs. TPA: $7.74 \mathrm{~ms}$ ). All two-way interactions were also not significant (Agent Type $\times$ Session: $F(1,70)=.61, p=.43, \eta^{2}<.001 ;$ Agent Type $\times$ Brain Site: $(F(1$, 
$\left.70)=2.24, p=.13, \eta^{2}<.01\right) ;$ Session $\times$ Brain Site: $\left(F(1,70)=.17, p=.67, \eta^{2}<.001\right)$. Most importantly, however, the three-way interaction of Agent Type $\times$ Session x Brain Site was significant $\left(F(1,70)=4.50, p=.03, \eta^{2}=.12\right)$ indicating that tDCS stimulation affected gaze cueing effects differently for the human versus the robot condition under left PFA but not left TPA stimulation.

To examine the significant three-way interaction effect further, two $2 \times 2$ post-hoc ANOVAs with Agent Type (Human vs. Robot) and Session (Baseline vs. Stimulation) were conducted, one for left PFA stimulation and one for left TPA stimulation. The ANOVA for the PFA stimulation condition showed no main effects of Agent Type $(F(1,35)=2.21, p=.14$, $\left.\eta^{2}=.01\right)$ or Session $\left(F(1,35)=.08, p=.77, \eta^{2}<.001\right)$, but a significant Agent Type $\times$ Session interaction $\left(F(1,35)=5.51, p=.02, \eta^{2}=.02\right)$. Post-hoc paired $t$-tests revealed that there was no significant difference in gaze cueing effects between the human and robot agents at baseline (Human: 7.47 ms vs. Robot: $8.66 \mathrm{~ms} ; \mathrm{p}=.75$ ), but significantly larger cueing effects for the human versus the robot gazer under stimulation (Human: 12.25 ms vs. Robot: $2.34 \mathrm{~ms} ; \mathrm{p}=$ .02). In contrast, the ANOVA for the TPA stimulation condition revealed neither main effects of Agent Type $\left(\mathrm{F}(1,35)=.47, \mathrm{p}=.49, \mathrm{\eta}^{2}<.01\right)$ or Session $\left(\mathrm{F}(1,35)=.09, \mathrm{p}=.76, \mathrm{\eta}^{2}<.01\right)$, nor a significant Agent Type $x$ Session interaction $\left(F(1,35)=.72, p=.39, \eta^{2}<.01\right)$. This suggests that while PFA stimulation modulated gaze cueing effects with significantly larger gaze-cueing effects for the human versus the robot agent under stimulation, TPA stimulation did not have such a modulatory effect on gaze cueing (i.e., no differences in gaze cueing at baseline and under stimulation). Post-hoc t-tests were corrected using the false discovery rate procedure (FDR). 
A separate $2 \times 2 \times 2$ mixed ANOVA was conducted on accuracy ratings of participants.

The ANOVA revealed no significant main effects or interaction effects. See the Behavioral

Results section of the Supplementary Materials for inference statistics. All data and materials can be publicly viewed on https://osf.io/s8ewg/.

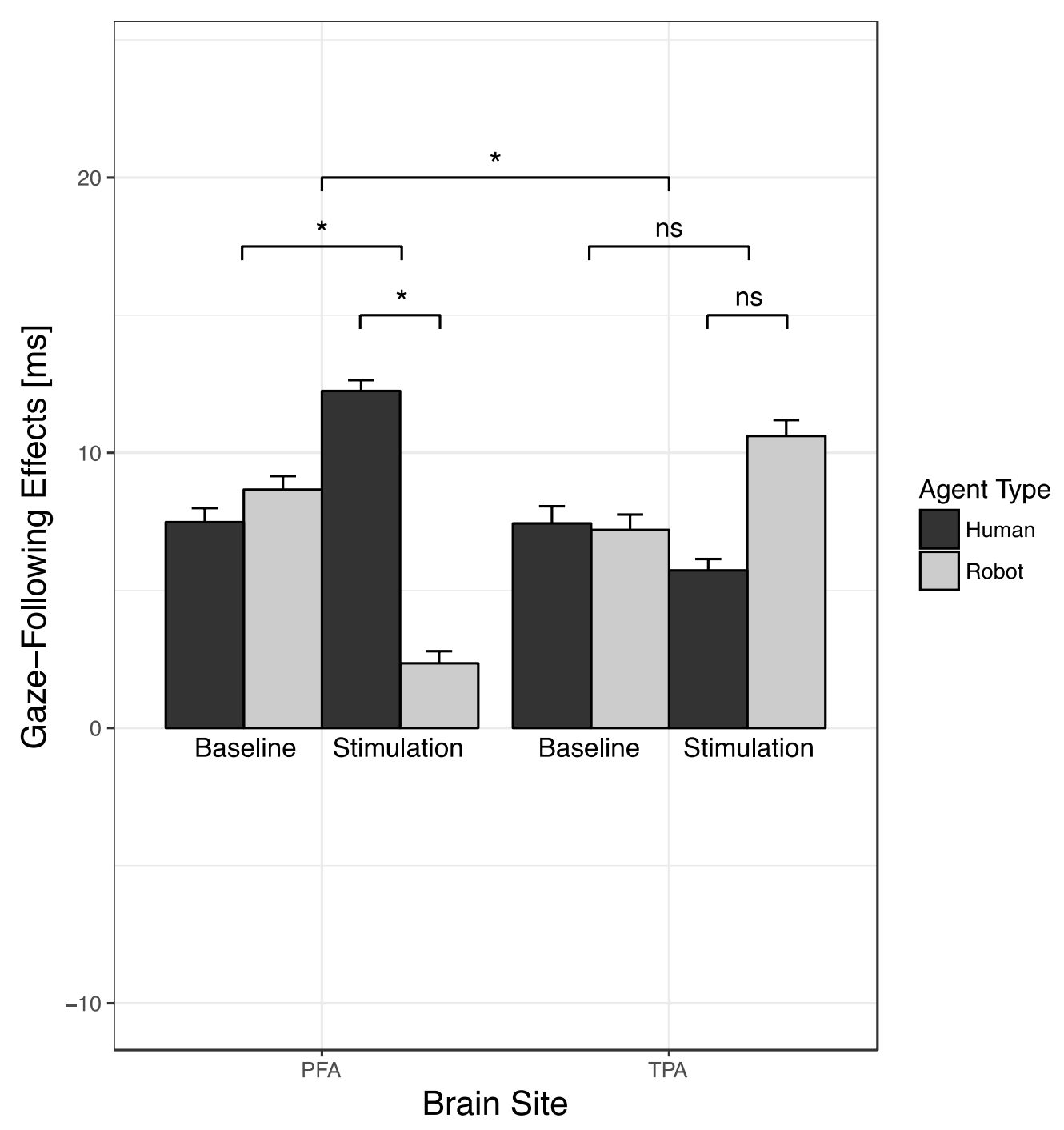

Figure 5. Gaze-cueing effects (in ms) as a function of Brain Site (left PFA, left TPA), Session (Baseline, Stimulation), and Agent Type (Human, Robot). There was a significant change in gaze cueing for active PFA stimulation, with no differences in gaze cueing between human and robot at baseline, but significantly larger gaze-cueing effects for the human versus the robot agent under stimulation. Active TPA stimulation did not have significant effects on gaze cueing $(* p<.05)$. 


\section{DISCUSSION}

Previous studies have shown that activation in left prefrontal $[10,31,43,57,110,111]$ and temporo-parietal $[25,27,43,90]$ areas is related to mind perception and the modulation of lowlevel social-cognitive processes like gaze cueing $[44,57,58]$. The goal of the current experiment was to examine the causal involvement of left prefrontal and left temporo-parietal areas in the top-down modulation of social attention via mind perception. To address this issue, we asked participants to perform a gaze-cueing task with a human and a robot agent (i.e., manipulation of mind perception via physical human-likeness) while applying tDCS to left prefrontal and left temporo-parietal areas. The findings show that stimulating left prefrontal had a modulatory effect on social attention, such that gaze cues of intentional agents (i.e., human) were followed significantly more strongly than gaze cues of machine agents (i.e., robot) under stimulation of prefrontal areas. Left temporo-parietal stimulation, in contrast, did not significantly modulate gaze-cueing effects for either of the two agents. These results are in line with previous studies showing that experimental manipulations of mind perception enhance the degree to which gaze signals are followed $[44,54,55]$. In particular, it was shown that interpreting gaze signals as intentional or human-controlled augments sensory processing of stimuli presented at gazed-at locations [54], and increases the social relevance of observed gaze signals $[53,55,79,112]$. The results are also in line with studies showing a correlational relationship between activation in left prefrontal areas related to mind perception and modulation of social attention when performing an orthogonal gaze cueing task outside the fMRI scanner [57], as well as tDCS studies showing that temporo-parietal areas are causally involved in social-cognitive processes 
like imitation and perspective taking but might not be causally involved in mind perception [113-115].

The current experiment adds to previous findings by localizing the source of top-down modulation of social attention via mind perception to left prefrontal areas, including areas like the ACC and vmPFC. These areas are associated with mentalizing [14,31], and involved in impression formation in social interaction $[116,117]$. In particular, activation in MPFC is linked to retrieving stereotypical knowledge about other people [118-120], and associated with retrieving script-based social knowledge $[121,122]$. Medial prefrontal areas are also more strongly activated when mentalizing about the internal states of similar than dissimilar others [117,123-125], as well as when viewing social scenes that contain human versus nonhuman agents [126]. The current experiment adds to the literature by indicating that prefrontal areas might not only is involved in the modulation of higher-order social-cognitive processes like decision-making $[31,43]$, but might also exert modulatory effects on low-level social cognitive processes like social attention.

Please note that the current experiment does not show the previously reported difference in gaze cueing between human and robot gazer at baseline [55]. This can be due to several reasons: First, in previous experiments, the gazers were introduced as "human" versus "robot" via instruction, which provided participants with explicit labels as to how to treat them in terms of mind perception (i.e., "human/has a mind" and "robot/has no mind"). In contrast, in the current experiment, the gazers were introduced more neutrally as "agents" and their mind status had to be inferred from physical appearance, which makes the mind perception manipulation more implicit. Although this certainly increases the external validity of the 
experimental manipulation, it is possible that participants did not pay enough attention to the gazers' mind status, which could wash out effects between the two agents at baseline. Second, since mind perception was manipulated via physical appearance in the current experiment, it is also possible that individual differences in anthropomorphism $[89,90]$ attenuated differences in gaze cueing between the human and robot gazer at baseline. However, since baseline effects are comparable for both stimulation conditions and since the current paper is mainly interested in the modulation of social attention via mind perception, insignificant differences in gaze cueing at baseline should not have impacted the reported findings. Nevertheless, in order to validate the robustness of the reported findings, future experiments should be conducted to determine to which degree they might be influenced by individual differences in anthropomorphism.

The question remains why stimulation of left temporo-parietal networks did not significantly modulate low-level mechanisms of social cognition despite previous reports showing a correlational relationship between mechanisms of social attention and bilateral TPJ activation [44]. One explanation for the lack of a significant effect of left temporo-parietal stimulation on gaze cueing is that it is possible that processes related to mind perception and social attention are not sufficiently interconnected at the level of the left TPJ in order to exert a top-down modulatory effect on attentional orienting to gaze cues. This interpretation would be in line with previous reports showing that social functions within the TPJ are lateralized [17], and that an overlap between attentional orienting and mentalizing is found within the right but not left TPJ $[11,127,128]$. In contrast, left TPJ lesions have been shown to cause selective deficits in false belief reasoning $[89,91]$, which does require mentalizing but no orientation of 
social attention. In support of this notion, it has been shown that early posterior ERP components like the N170 are sensitive to the intentionality of an agent without being responsive to the congruency or social outcome of its gaze cues [112], whereas later anterior ERP components like the P350 are sensitive to both an agent's intentionality and the congruency of gaze cues [78] (i.e., significant difference in P350 amplitudes for invalid vs. valid trials for human vs. computer-controlled conditions), suggesting that the integration of mind perception related processes and social attention might be instantiated in prefrontal (but not temporo-parietal) areas.

Another possible explanation is that prefrontal and temporo-parietal brain regions might process information about an agent's mind on different levels, with TPJ activation being related to inferring particular internal states from observed behaviors $[11,19,27,110]$ (e.g., observing an agent smile leads to the inference that the agent is currently in a state of happiness), and MPFC and VMPFC activation being related to reasoning based on stereotypical assumptions regarding general traits associated with intentional agents [129] (e.g., agent that looks like a child might like toys). It would be possible that studies that manipulate mind perception via instruction of particular beliefs (i.e., "eye movements are intentional") engage the posterior mind perception network [44], while studies that manipulate mind perception via physical appearance (i.e., the agent looks human) more strongly activate stereotypical assumptions about human behavior, thereby engaging the anterior mind perception network involving the MPFC and VMPFC [118-120]. This interpretation is particularly plausible given that attentional orienting to gaze cues is a fast-acting process [67], which requires information from a readily available source in order to be top-down controlled. Since stereotypical 
information about an agent is more readily available than the outcomes of mentalizing processes about particular internal states, a stronger involvement of prefrontal areas in the top-down modulation of fast processes like social attention seems tenable.

A third explanation is that the observed modulation of gaze cueing is not specific to mind perception, but due to other (related) functions associated with the left prefrontal cortex. One aspect of the experimental design that could have affected social attention in addition to the gazer's physical appearance is the non-predictivity of gaze cues in the current experiment (i.e., targets appear with equal frequency at validly and invalidly cued locations). Previous studies have shown that stimuli whose behavior is hard to predict are more likely anthropomorphized, and that evaluating unpredictable stimuli is associated with increased activation in medial prefrontal areas, and specifically the VMPFC and ACC [49]. In consequence, it is possible that one's sensitivity to the predictability of gaze cues had an impact on the degree to which the gazer was anthropomorphized and prefrontal brain areas were activated during gaze cueing. For the current experiment, this means that stimulating prefrontal areas may have specifically enhanced the social relevance of human gaze cues, leading to longer processing times on invalid trials [78] and larger gaze-cueing effects (i.e., difference in reaction times between invalid and valid trials), while temporo-parietal stimulation may not have affected the perceived social relevance of human gaze cues².

Alternatively, the VMPFC has been shown to track feelings of eeriness towards nonhuman agents in a parametric fashion [129] and has been labeled the potential neural

\footnotetext{
2 Please note that the conducted a-priori power analysis was based on a small-to-medium effect size, which leaves the possibility that very small-to-small effects might not have been detected with the current sample size.
} 
correlate of the uncanny valley [130] (i.e., nonhuman agents with human-like appearance induce feelings of eeriness if they are not perfectly human). If that were the case, stimulation of prefrontal areas could have enhanced feelings of eeriness towards the robotic agent, leading to a disengagement from robot gaze cues together with an increased engagement in attending to human gaze cues (i.e., eeriness of robot cues made human gaze cues more "desirable"). Effects related to non-social prefrontal functions such as working memory, executive functioning or abstract reasoning are less likely to have influenced gaze cueing, since one would expect comparable effects of stimulation for human and nonhuman agents. Whether prefrontal stimulation modulated gaze cueing directly via mind perception or via processes affected by mind perception, such as perception of uncertainty [78] or emotional reactions to uncanny agents [129], cannot ultimately be answered based on the current data and requires follow-up studies. It can also not be clearly determined - due to the lack of spatial specificity of tDCS which prefrontal brain area(s) ultimately caused the observed top-down modulation of social attention (i.e., areas directly implicated in mind perception such as the left ACC, or areas that are indirectly involved in mind perception such as the left MPFC, VMPFC or DLPFC).

\section{CONCLUSIONS}

Previous studies have shown that the degree to which we attend to social cues depends on the degree to which we perceive mind in the entity sending the cues. The neural correlates of mind perception have been localized to prefrontal and temporo-parietal structures in previous studies, but the causal involvement of these areas in the modulation of low-level socialcognitive processes like gaze cueing has not been determined yet. The current study shows that 
stimulation to prefrontal areas increases the degree to which human gaze is followed compared to the degree to which robot gaze is followed, while stimulation to temporo-parietal regions does not seem to have a measurable modulatory effect on gaze cueing. Since the effect of prefrontal stimulation is only observable for human gazers, it is tenable that prefrontal stimulation does not simply lead someone to perceive "more" mind in others, but rather seems to enhance the social relevance of signals coming from agents "with a mind". In other words, prefrontal stimulation does not seem to make participants perceive more human-likeness in non-human agents, which makes it unlikely that the observed effect is related to anthropomorphism. Instead, prefrontal stimulation seems to help discriminate agents "with a mind" from agents "without a mind", as evidenced by an increased difference in gaze cueing between the human and the robot gazer under stimulation, indicating that stimulation of prefrontal areas enhances the importance of social signals coming from human agents. Taken together, this study shows a causal link between prefrontal stimulation and mechanisms of social attention, and dissociation between the anterior and posterior part of the social brain network in terms of top-down modulation of social-cognitive processes. Whether the effect is specific to mind perception or related to processes indirectly affected by mind perception needs to be determined in future studies.

\section{REFERENCES}

1. Tapus A, Matarić M. Towards socially assistive robots. J Robot Soc Jpn. 2006; 14(5):5768.

2. Scassellati B, Admoni H, Matarić M. Robots for Use in Autism Research. Annu Rev Biomed Eng. 2012; 14:275-94.

3. Basteris A, Nijenhuis SM, Stienen AH, Buurke JH, Prange GB, Amirabdollahian F. Training modalities in robot-mediated upper limb rehabilitation in stroke: a framework for classification based on a systematic review. J NeuroEngineering Rehabil. 2014;11(1):111. 
4. Mubin O, Stevens CJ, Shahid S, Mahmud AA, Dong J-J. A REVIEW OF THE APPLICABILITY OF ROBOTS IN EDUCATION. Technol Educ Learn [Internet]. 2013 [cited 2018 Nov 5];1(1). Available from: http://www.actapress.com/PaperInfo.aspx?paperld=43268

5. Bartneck C, Reichenbach J. Subtle emotional expressions of synthetic characters. Int J Hum-Comput Stud. 2005 Feb;62(2):179-92.

6. Scopelliti M, Giuliani MV, Fornara F. Robots in a domestic setting: a psychological approach. Univers Access Inf Soc. 2005 Dec;4(2):146-55.

7. Baron-Cohen S. Mindblindness: An essay on autism and theory of mind. MIT press; 1997.

8. Frith $\mathrm{CD}$, Frith $\mathrm{U}$. How we predict what other people are going to do. Brain Res. 2006;1079:36-46.

9. Adolphs R. The Social Brain: Neural Basis of Social Knowledge. Annu Rev Psychol. 2009;60(1):693-716.

10. Van Overwalle F, Baetens K. Understanding others' actions and goals by mirror and mentalizing systems: A meta-analysis. Neurolmage. 2009;48(3):564-84.

11. Bzdok D, Langner R, Schilbach L, Engemann DA, Laird AR, Fox PT, et al. Segregation of the human medial prefrontal cortex in social cognition. Front Hum Neurosci [Internet]. 2013;7. Available from: http://journal.frontiersin.org/article/10.3389/fnhum.2013.00232/abstract

12. Spunt RP, Adolphs R. Validating the Why/How contrast for functional MRI studies of Theory of Mind. Neurolmage. 2014;99:301-11.

13. Spunt RP, Lieberman MD. Dissociating Modality-Specific and Supramodal Neural Systems for Action Understanding. J Neurosci. 2012;32(10):3575-83.

14. Amodio DM, Frith CD. Meeting of minds: the medial frontal cortex and social cognition. Nat Rev Neurosci. 2006 Apr;7(4):268-77.

15. Saxe R, Carey S, Kanwisher N. Understanding other minds: linking developmental psychology and functional neuroimaging. Annu Rev Psychol. 2004;55:87-124.

16. Gallagher HL, Frith CD. Functional imaging of 'theory of mind.' Trends Cogn Sci. 2003;7(2):77-83.

17. Perner J, Aichhorn M, Kronbichler M, Staffen W, Ladurner G. Thinking of mental and other representations: The roles of left and right temporo-parietal junction. Soc Neurosci. 2006;1(3-4):245-58.

18. Grèzes J, Berthoz S, Passingham RE. Amygdala activation when one is the target of deceit: Did he lie to you or to someone else? Neurolmage. 2006;30(2):601-8.

19. Saxe R, Powell LJ. It's the thought that counts: Specific brain regions for one component of theory of mind. Psychol Sci. 2006;17(8):692-9.

20. Ohnishi T, Moriguchi Y, Matsuda H, Mori T, Hirakata M, Imabayashi E, et al. The neural network for the mirror system and mentalizing in normally developed children: An fMRI study. NeuroReport. 2004;15(9):1483-7.

21. Grezes J. Brain Mechanisms for Inferring Deceit in the Actions of Others. J Neurosci. 2004;24(24):5500-5.

22. Farrer C, Franck N, Georgieff N, Frith CD, Decety J, Jeannerod M. Modulating the experience of agency: A positron emission tomography study. Neurolmage. 2003;18(2):324-33. 
23. Saxe R, Kanwisher N. People thinking about thinking people: The role of the temporoparietal junction in "theory of mind." Neurolmage. 2003;1835-42.

24. Chaminade T, Decety J. Leader or follower? Involvement of the inferior parietal lobule in agency. Neuroreport. 2002;13(15):1975-8.

25. Gallagher HL, Happé F, Brunswick N, Fletcher PC, Frith U, Frith CD. Reading the mind in cartoons and stories: An fMRI study of "theory of mind" in verbal and nonverbal tasks. Neuropsychologia. 2000;38(1):11-21.

26. Ruby $P$, Decety J. Effect of subjective perspective taking during simulation of action: a PET investigation of agency. Nat Neurosci. 2001;4(5):546-50.

27. Van Overwalle F. Social cognition and the brain: A meta-analysis. Hum Brain Mapp. 2009;30(3):829-58.

28. Saxe R. Uniquely human social cognition. Curr Opin Neurobiol. 2006;16(2):235-9.

29. Saxe R, Wexler A. Making sense of another mind: The role of the right temporo-parietal junction. Neuropsychologia. 2005;43(10):1391-9.

30. McCabe K, Houser D, Ryan L, Smith V, Trouard T. A functional imaging study of cooperation in two-person reciprocal exchange. Proc Natl Acad Sci. 2001;98(20):118325.

31. Gallagher HL, Jack Al, Roepstorff A, Frith CD. Imaging the intentional stance in a competitive game. Neurolmage. 2002;16:814-21.

32. Spunt RP, Meyer ML, Lieberman MD. The Default Mode of Human Brain Function Primes the Intentional Stance. J Cogn Neurosci. 2015 Jun;27(6):1116-24.

33. Gray HM, Gray K, Wegner DM. Dimensions of Mind Perception. Science. 2007;315(5812):619-619.

34. Epley N, Waytz A, Cacioppo JT. On Seeing Human: A Three-Factor Theory of Anthropomorphism. Psychol Rev. 2007;114(4):864-86.

35. Kiesler S, Powers A, Fussell SR, Torrey C. Anthropomorphic interactions with a robot and robot-like agent. Soc Cogn. 2008;26(2):169-181.

36. DiSalvo C, Gemperle F. From Seduction to Fulfillment: The Use of Anthropomorphic Form in Design. Int Conf Des Pleasurable Prod Interfaces. 2003;67-72.

37. Castelli F, Happé F, Frith U, Frith C. Movement and Mind: A Functional Imaging Study of Perception and Interpretation of Complex Intentional Movement Patterns. Neurolmage. 2000 Sep;12(3):314-25.

38. Heider F, Simmel M. An Experimental Study of Apparent Behavior. Am J Psychol. 1944;57(2):243-59.

39. Gutsell JN, Inzlicht M. Intergroup differences in the sharing of emotive states: Neural evidence of an empathy gap. Soc Cogn Affect Neurosci. 2012;7(5):596-603.

40. Saygin AP, Chaminade T, Ishiguro $H$, Driver J, Frith $C$. The thing that should not be: predictive coding and the uncanny valley in perceiving human and humanoid robot actions. SCAN. 2012;7:413-22.

41. Harris LT, Fiske ST. Perceiving Humanity or Not: A Social Neuroscience Approach to Dehumanized Perception. In: Social Neuroscience: Toward Understanding the Underpinnings of the Social Mind. 2011. 
42. Cehajic S, Brown R, Gonzalez R. What do i care? Perceived ingroup responsibility and dehumanization as predictors of empathy felt for the victim group. Group Process Intergroup Relat. 2009;12(6):715-29.

43. Sanfey AG, Rilling JK, Aronson JA, Nystrom LE, Cohen JD. The neural basis of economic decision-making in the Ultimatum Game. Science. 2003;300(5626):1755-8.

44. Özdem C, Wiese E, Wykowska A, Müller H, Brass M, Van Overwalle F. Believing androids - $\mathrm{fMRI}$ activation in the right temporo-parietal junction is modulated by ascribing intentions to non-human agents. Soc Neurosci. 2016;12(5):582-93.

45. Haley KJ, Fessler DMT. Nobody's watching? Subtle cues affect generosity an anonymous economic game. Evol Hum Behav. 2005;26(3):245-56.

46. Bering JM, Johnson D. " O Lord... You Perceive my Thoughts from Afar": Recursiveness and the evolution of supernatural agency. J Cogn Cult. 2005;5(1):118-42.

47. Hertz N, Wiese E. Social Facilitation with Non-Human Agents: Possible or not? Proc Hum Factors Ergon Soc Annu Meet. 2017 Sep;61(1):222-5.

48. Riether N, Hegel F, Wrede B, Horstmann G. Social facilitation with social robots? In: Proceedings of the seventh annual ACM/IEEE international conference on Human-Robot Interaction - HRI '12 [Internet]. Boston, Massachusetts, USA: ACM Press; 2012 [cited 2018 Aug 15]. p. 41. Available from: http://dl.acm.org/citation.cfm?doid=2157689.2157697

49. Waytz A, Gray K, Epley N, Wegner DM. Causes and consequences of mind perception. Trends Cogn Sci. 2010 Aug;14(8):383-8.

50. Short E, Hart J, Vu M, Scassellati B. No fair!! An interaction with a cheating robot. 2010 5th ACMIEEE Int Conf Hum-Robot Interact HRI. 2010;219-26.

51. Takahashi K, Watanabe K. Gaze Cueing by Pareidolia Faces. -Percept. 2013 Dec;4(8):4902.

52. Abubshait A, Wiese E. You Look Human, But Act Like a Machine: Agent Appearance and Behavior Modulate Different Aspects of Human-Robot Interaction. Front Psychol [Internet]. 2017 Aug 23;8. Available from: http://journal.frontiersin.org/article/10.3389/fpsyg.2017.01393/full

53. Caruana N, de Lissa $P$, McArthur G. Beliefs about human agency influence the neural processing of gaze during joint attention. Soc Neurosci. 2016;12(2):194-206.

54. Wykowska A, Wiese E, Prosser A, Müller HJ. Beliefs about the Minds of Others Influence How We Process Sensory Information. Hamed SB, editor. PLoS ONE. 2014 Apr 8;9(4):e94339.

55. Wiese E, Wykowska A, Zwickel J, Müller HJ. I See What You Mean: How Attentional Selection Is Shaped by Ascribing Intentions to Others. Hamed SB, editor. PLoS ONE. 2012 Sep 26;7(9):e45391.

56. Epley N, Waytz A, Akalis S, Cacioppo JT. When We Need A Human: Motivational Determinants of Anthropomorphism. Soc Cogn. 2008;26(2):143-55.

57. Wiese E, Buzzell GA, Abubshait A, Beatty PJ. Seeing minds in others: Mind perception modulates low-level social-cognitive performance and relates to ventromedial prefrontal structures. Cogn Affect Behav Neurosci. 2018 Oct;18(5):837-56.

58. Wiese E, Metta G, Wykowska A. Robots As Intentional Agents: Using Neuroscientific Methods to Make Robots Appear More Social. Front Psychol. 2017;8(October):1663. 
59. Coffman BA, Clark VP, Parasuraman R. Battery powered thought: Enhancement of attention, learning, and memory in healthy adults using transcranial direct current stimulation. Neurolmage. 2014 Jan;85:895-908.

60. Jacobson L, Koslowsky M, Lavidor M. TDCS polarity effects in motor and cognitive domains: A meta-analytical review. Exp Brain Res. 2012;216(1):1-10.

61. Antal A, Nitsche MA, Paulus W. External modulation of visual perception in humans. Neuroreport. 2001;12(16):3553-5.

62. Cohen Kadosh R, Soskic S, luculano T, Kanai R, Walsh V. Modulating neuronal activity produces specific and long-lasting changes in numerical competence. Curr Biol. 2010;20(22):2016-20.

63. Nummenmaa L, Calder AJ. Neural mechanisms of social attention. Trends Cogn Sci. 2009 Mar;13(3):135-43.

64. Adams RB, Kleck RE. Effects of direct and averted gaze on the perception of facially communicated emotion. Emotion. 2005;5(1):3-11.

65. Blakemore SJ, Winston J, Frith U. Social cognitive neuroscience: where are we heading? Trends Cogn Sci. 2004;8(5):216-22.

66. Frischen A, Bayliss AP, Tipper SP. Gaze cueing of attention: Visual attentionn, Social Cognition, and Individual Differences. Psychol Bull. 2007;133(4):694-724.

67. Friesen $\mathrm{CK}$, Kingstone $\mathrm{A}$. The eyes have it! Reflexive orienting is triggered by nonpredictive gaze. Psychon Bull Rev. 1998;5(3):490-5.

68. Dalmaso M, Edwards SG, Bayliss AP. Re-encountering individuals who previously engaged in joint gaze modulates subsequent gaze cueing. J Exp Psychol Learn Mem Cogn. 2016;42(2):271-84.

69. Cazzato V, Liuzza MT, Caprara GV, Macaluso E, Aglioti SM. The attracting power of the gaze of politicians is modulated by the personality and ideological attitude of their voters: A functional magnetic resonance imaging study. Eur J Neurosci. 2015;42(8):253445.

70. Porciello G, Holmes BS, Liuzza MT, Crostella F, Aglioti SM, Bufalari I. Interpersonal Multisensory Stimulation reduces the overwhelming distracting power of self-gaze: psychophysical evidence for 'engazement.' Sci Rep. 2014;4(6669):1-7.

71. Ciardo F, Marino BFM, Actis-Grosso R, Rossetti A, Ricciardelli P. Face age modulates gaze following in young adults. Sci Rep. 2014;4:4746.

72. Hungr $\mathrm{CJ}$, Hunt AR. Physical self-similarity enhances the gaze-cueing effect. Q J Exp Psychol. 2012;65(7):1250-9.

73. Kawai N. Attentional shift by eye gaze requires joint attention: Eye gaze cues are unique to shift attention1: Social attention by the gaze cues. Jpn Psychol Res. 2011 Sep;53(3):292-301.

74. Bonifacci P, Ricciardelli P, Lugli L, Pellicano A. Emotional attention: effects of emotion and gaze direction on overt orienting of visual attention. Cogn Process. 2008 May;9(2):127-35.

75. Fox MD, Snyder AZ, Vincent JL, Raichle ME. Intrinsic Fluctuations within Cortical Systems Account for Intertrial Variability in Human Behavior. Neuron. 2007;56(1):171-84.

76. Tipples J. Fear and fearfulness potentiate automatic orienting to eye gaze. Cogn Emot. 2006;20(2):309-20. 
77. Ristic J, Kingstone A. Taking control of reflexive social attention. Cognition. 2005 Jan;94(3):B55-65.

78. Caruana N, McArthur G, Woolgar A, Brock J. Simulating social interactions for the experimental investigation of joint attention. Neurosci Biobehav Rev. 2017 Mar;74:11525.

79. Wiese E, Wykowska A, Müller HJ. What We Observe Is Biased by What Other People Tell Us: Beliefs about the Reliability of Gaze Behavior Modulate Attentional Orienting to Gaze Cues. Hamed SB, editor. PLoS ONE. 2014 Apr 10;9(4):e94529.

80. Teufel C, Alexis DM, Todd H, Lawrance-Owen AJ, Clayton NS, Davis G. Social Cognition Modulates the Sensory Coding of Observed Gaze Direction. Curr Biol. 2009 Aug;19(15):1274-7.

81. Rosenthal-Von Der Pütten AM, Krämer NC. How design characteristics of robots determine evaluation and uncanny valley related responses. Comput Hum Behav. 2014;36:422-39.

82. Bartneck C. Robots in the theatre and the media. Des Semant Form Mov DeSForM2013. 2013;64-70.

83. Jack Al, Robbins P. The Phenomenal Stance Revisited. Rev Philos Psychol. 2012;3(3):383403.

84. Krach S, Hegel F, Wrede B, Sagerer G, Binkofski F, Kircher T. Can machines think? Interaction and perspective taking with robots investigated via fMRI. PLoS ONE. 2008;3(7).

85. Takahashi H, Terada K, Morita T, Suzuki S, Haji T, Kozima H, et al. Different impressions of other agents obtained through social interaction uniquely modulate dorsal and ventral pathway activities in the social human brain. Cortex. 2014;58:289-300.

86. Gobbini MI, Gentili C, Ricciardi E, Bellucci C, Salvini P, Laschi C, et al. Distinct Neural Systems Involved in Agency and Animacy Detection. J Cogn Neurosci. 2011;23(8):191120.

87. Carter EJ, Hodgins JK, Rakison DH. Exploring the neural correlates of goal-directed action and intention understanding. Neurolmage. 2011;54(2):1634-42.

88. Gobel MS, Tufft MRA, Richardson DC. Social Beliefs and Visual Attention: How the Social Relevance of a Cue Influences Spatial Orienting. Cogn Sci. 2017 May;42:161-85.

89. Hackel LM, Looser CE, Van Bavel JJ. Group membership alters the threshold for mind perception: The role of social identity, collective identification, and intergroup threat. Journal of Experimental Social Psychology. 2014;52:15-23.

90. Cullen $\mathrm{H}$, Kanai R, Bahrami B, Rees $\mathrm{G}$. Individual differences in anthropomorphic attributions and human brain structure. Sc Cogn Affect Neurosci. 2013;9(9):1276-80.

91. Apperly IA, Samson D, Chiavarino C, Humphreys GW. Frontal and Temporo-Parietal Lobe Contributions to Theory of Mind: Neuropsychological Evidence from a False-Belief Task with Reduced Language and Executive Demands. J Cogn Neurosci. 2004;16(10):1773-84.

92. Polanía R, Nitsche MA, Ruff CC. Studying and modifying brain function with non-invasive brain stimulation. Nat Neurosci. 2018 Feb;21(2):174-87.

93. Parkin BL, Ekhtiari H, Walsh VF. Non-invasive Human Brain Stimulation in Cognitive Neuroscience: A Primer. Neuron. 2015;87(5):932-45. 
94. Bikson M, Datta A, Elwassif M. Establishing safety limits for transcranial direct current stimulation. Clin Neurophysiol. 2009 Jun;120(6):1033-4.

95. Oostenveld R, Praamstra P. The five percent electrode system for high-resolution EEG and ERP measurements. Clin Neurophysiol. 2001;7.

96. Blumberg EJ, Foroughi CK, Scheldrup MR, Peterson MS, Boehm-Davis DA, Parasuraman R. Reducing the Disruptive Effects of Interruptions With Noninvasive Brain Stimulation. Hum Factors. 2014;57(6):1051-62.

97. Falcone B, Coffman BA, Clark VP, Parasuraman R. Transcranial Direct Current Stimulation Augments Perceptual Sensitivity and 24-Hour Retention in a Complex Threat Detection Task. PLoS ONE. 2012;7(4):e34993.

98. Datta A, Truong D, Minhas P, Parra LC, Bikson M. Inter-Individual Variation during Transcranial Direct Current Stimulation and Normalization of Dose Using MRI-Derived Computational Models. Front Psychiatry [Internet]. 2012 [cited 2018 Apr 6];3. Available from: http://journal.frontiersin.org/article/10.3389/fpsyt.2012.00091/abstract

99. Moliadze V, Antal A, Paulus W. Electrode-distance dependent after-effects of transcranial direct and random noise stimulation with extracephalic reference electrodes. Clin Neurophysiol. 2010 Dec;121(12):2165-71.

100. Bartneck C, Croft E, Kulic D. Measuring the anthropomorphism, animacy, likeability, perceived intelligence, and perceived safety of robots. Metr HRI Workshop Tech Rep. 2008;

101. Nomura T, Kanda T, Suzuki T. Experimental investigation into influence of negative attitudes toward robots on human-robot interaction. AI Soc. 2006;20(2):138-50.

102. Baron-Cohen S, Wheelwright S, Skinner R, Martin J, Clubley E. The Autism-Spectrum Quotient (AQ): Evidence from Asperger Syndrome/High-Functioning Autism, Males and Females, Scientists and Mathematicians. J Autism Dev Disord. 2001;31(1):5-17.

103. Javadi $A H$, Cheng $P$, Walsh V. Short duration transcranial direct current stimulation (tDCS) modulates verbal memory. Brain Stimulat. 2012 Oct;5(4):468-74.

104. Berryhill ME. Hits and misses: leveraging tDCS to advance cognitive research. Front Psychol [Internet]. 2014 [cited 2018 Aug 20];5. Available from: http://journal.frontiersin.org/article/10.3389/fpsyg.2014.00800/abstract

105. Boggio PS, Rocha RR, da Silva MT, Fregni F. Differential modulatory effects of transcranial direct current stimulation on a facial expression go-no-go task in males and females. Neurosci Lett. 2008 Dec;447(2-3):101-5.

106. Ferrucci R, Mameli F, Guidi I, Mrakic-Sposta S, Vergari M, Marceglia S, et al. Transcranial direct current stimulation improves recognition memory in Alzheimer disease. Neurology. 2008 Aug 12;71(7):493-8.

107. Nitsche MA, Cohen LG, Wassermann EM, Priori A, Lang N, Antal A, et al. Transcranial direct current stimulation: State of the art 2008. Brain Stimulat. 2008;1(3):206-23.

108. Rani Das K, Imon, Rahmatullah. A Brief Review of Tests for Normality. Am J Theor Appl Stat. 2016;5(1):5.

109. Park YG. Comments on Statistical Issues in January 2013. Korean J Fam Med. 2013;34(1):64.

110. Schurz M, Radua J, Aichhorn M, Richlan F, Perner J. Fractionating theory of mind: A metaanalysis of functional brain imaging studies. Neurosci Biobehav Rev. 2014;42:9-34. 
111. Chaminade T, Rosset D, Da Fonseca D, Nazarian B, Lutcher E, Cheng G, et al. How do we think machines think? An fMRI study of alleged competition with an artificial intelligence. Front Hum Neurosci [Internet]. 2012;6. Available from: http://journal.frontiersin.org/article/10.3389/fnhum.2012.00103/abstract

112. Caruana $\mathrm{N}$, de Lissa $\mathrm{P}, \mathrm{McArthur} \mathrm{G}$. The neural time course of evaluating self-initiated joint attention bids. Brain Cogn. 2015 Aug;98:43-52.

113. Santiesteban I, Banissy MJ, Catmur C, Bird G. Enhancing Social Ability by Stimulating Right Temporoparietal Junction. Curr Biol. 2012 Dec;22(23):2274-7.

114. Santiesteban I, Banissy MJ, Catmur C, Bird G. Functional lateralization of temporoparietal junction - imitation inhibition, visual perspective-taking and theory of mind. Thut $\mathrm{G}$, editor. Eur J Neurosci. 2015 Oct;42(8):2527-33.

115. Hogeveen J, Obhi SS, Banissy MJ, Santiesteban I, Press C, Catmur C, et al. Task-dependent and distinct roles of the temporoparietal junction and inferior frontal cortex in the control of imitation. Soc Cogn Affect Neurosci. 2015 Jul;10(7):1003-9.

116. Szczepanski SM, Knight RT. Insights into Human Behavior from Lesions to the Prefrontal Cortex. Neuron. 2014;83(5):1002-18.

117. Mitchell JP, Banaji MR, Macrae CN. General and specific contributions of the medial prefrontal cortex to knowledge about mental states. Neurolmage. 2005;28(4):757-62.

118. Fairhall SL, Anzellotti S, Ubaldi S, Caramazza A. Person- and place-selective neural substrates for entity-specific semantic access. Cereb Cortex. 2014;24(7):1687-96.

119. Contreras JM, Banaji MR, Mitchell JP. Dissociable neural correlates of stereotypes and other forms of semantic knowledge. Soc Cogn Affect Neurosci. 2012;7(7):764-70.

120. Simmons WK, Reddish M, Bellgowan PSF, Martin A. The selectivity and functional connectivity of the anterior temporal lobes. Cereb Cortex. 2010;20(4):813-25.

121. Ghosh VE, Moscovitch M, Melo Colella B, Gilboa A. Schema Representation in Patients with Ventromedial PFC Lesions. J Neurosci. 2014;34(36):12057-70.

122. Van Kesteren MTR, Ruiter DJ, Fern??ndez G, Henson RN. How schema and novelty augment memory formation. Trends Neurosci. 2012;35(4):211-9.

123. Jenkins AC, Macrae CN, Mitchell JP. Repetition suppression of ventromedial prefrontal activity during judgments of self and others. Proc Natl Acad Sci U S A.

2008;105(11):4507-12.

124. Mitchell JP. Encoding-Specific Effects of Social Cognition on the Neural Correlates of Subsequent Memory. J Neurosci. 2004 May 26;24(21):4912-7.

125. Mitchell JP, Macrae CN, Banaji MR. Dissociable Medial Prefrontal Contributions to Judgments of Similar and Dissimilar Others. Neuron. 2006;50(4):655-63.

126. Wagner DD, Kelley WM, Heatherton TF. Individual differences in the spontaneous recruitment of brain regions supporting mental state understanding when viewing natural social scenes. Cereb Cortex. 2011;21(12):2788-96.

127. Kubit B, Jack Al. Rethinking the role of the rTPJ in attention and social cognition in light of the opposing domains hypothesis: findings from an ALE-based meta-analysis and restingstate functional connectivity. Front Hum Neurosci [Internet]. 2013;7. Available from: http://journal.frontiersin.org/article/10.3389/fnhum.2013.00323/abstract 
128. Krall SC, Rottschy C, Oberwelland E, Bzdok D, Fox PT, Eickhoff SB, et al. The role of the right temporoparietal junction in attention and social interaction as revealed by ALE meta-analysis. Brain Struct Funct. 2015;220(2):587-604.

129. Wang Y, Quadflieg S. In our own image? Emotional and neural processing differences when observing human-human vs human-robot interactions. Soc Cogn Affect Neurosci. 2015;10(11):1515-24.

130. Mori M. The Uncanny Valley: The Original Essay by Masahiro Mori - IEEE Spectrum [Internet]. Energy. 1970. Available from: http://spectrum.ieee.org/automaton/robotics/humanoids/the-uncanny-valley

131. Balas B, Tonsager C. Face Animacy is Not All in the Eyes: Evidence from Contrast Chimeras. Perception. 2014 May;43(5):355-67.

132. Deska JC, Lloyd EP, Hugenberg K. Advancing Our Understanding of the Interface Between Perception and Intergroup Relations. Psychological Inquiry. 2016 Oct;27(4):286-9.

133. Gao T, McCarthy G, Scholl BJ. The Wolfpack Effect: Perception of Animacy Irresistibly Influences Interactive Behavior. Psychological Science. 2010 Dec;21(12):1845-53.

134. Schein C, Gray K. The Unifying Moral Dyad: Liberals and Conservatives Share the Same Harm-Based Moral Template. Personality and Social Psychology Bulletin. 2015 Aug;41(8):1147-63.

135. Looser CE, Wheatley T. The Tipping Point of Animacy: How, When, and Where We Perceive Life in a Face. Psychological Science. 2010 Dec 1;21(12):1854-62.

136. Wheatley T, Weinberg A, Looser C, Moran T, Hajcak G. Mind Perception: Real but Not Artificial Faces Sustain Neural Activity beyond the N170/VPP. Barnes S, editor. PLoS ONE. 2011 Mar 31;6(3):e17960.

137. Maurer D, Grand RL, Mondloch CJ. The many faces of configural processing. Trends in Cognitive Sciences. 2002 Jun;6(6):255-60.

138. Zink CF, Kempf L, Hakimi S, Rainey CA, Stein JL, Meyer-Lindenberg A. Vasopressin modulates social recognition-related activity in the left temporoparietal junction in humans. Translational Psychiatry. 2011 Apr;1(4):e3-e3.

139. Perner J, Aichhorn M, Kronbichler M, Staffen W, Ladurner G. Thinking of mental and other representations: The roles of left and right temporo-parietal junction. Social Neuroscience. 2006 Sep;1(3-4):245-58.

140. Chaminade T, Hodgins J, Kawato M. Anthropomorphism influences perception of computer-animated characters' actions. Social Cognitive and Affective Neuroscience. 2007 Sep;2(3):206-16.

141. Gallagher HL, Jack AI, Roepstorff A, Frith CD. Imaging the intentional stance in a competitive game. Neurolmage. 2002;16:814-21.

142. MacDorman KF, Entezari SO. Individual differences predict sensitivity to the uncanny valley. Interaction Studies. 2015;16(2):141-72. 


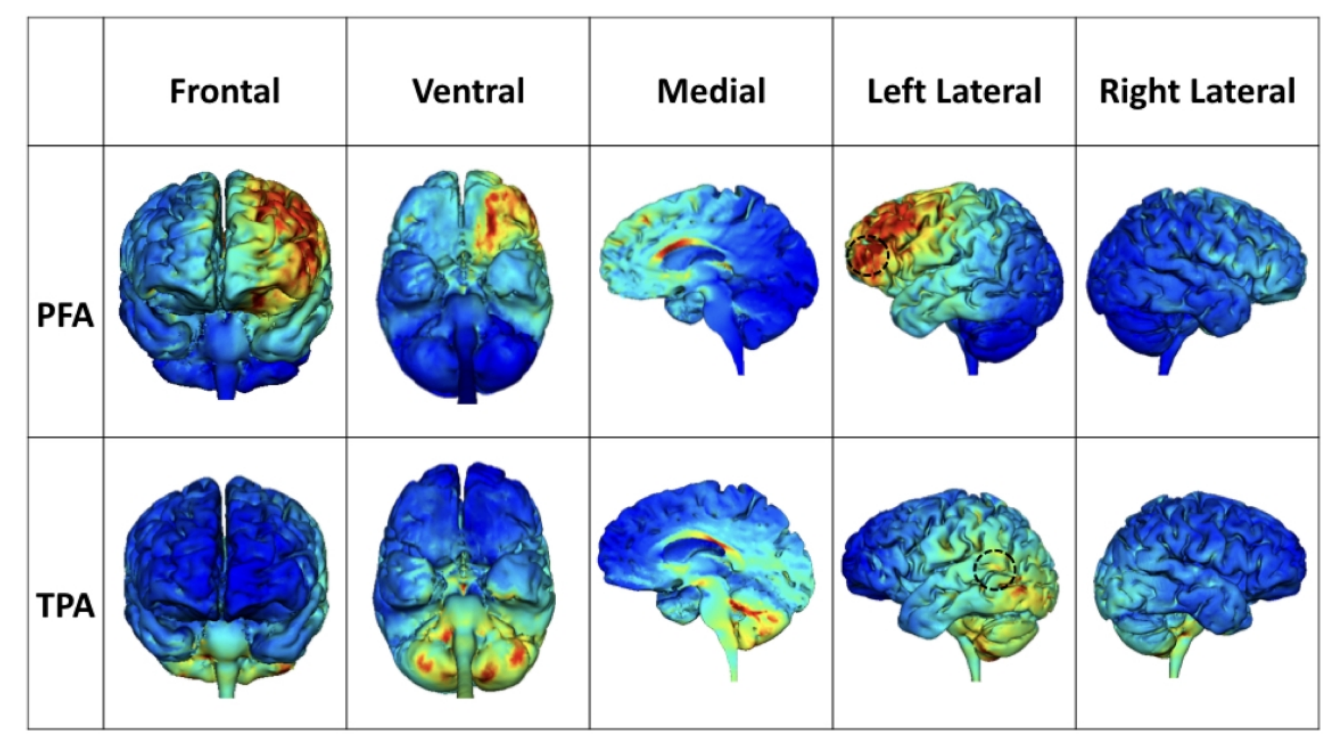

Figure 1. Brain Models illustrate the field intensities of stimulation in $\mathrm{V} / \mathrm{m}$ per $\mathrm{mA}$. Darker red areas illustrate higher stimulation intensities compared to blue areas. Please see Supplementary Materials for exact stimulation intensities of each structure.

\section{$338 \times 190 \mathrm{~mm}(300 \times 300$ DPI)}


Figure 2. Human and Robot Stimuli: the human agent is represented by a female face taken from the Karolinska Directed Emotional Faces (KDEF) database (F07; written informed consent from Karolinska Institute was received to use the photograph for experimental investigations and illustrations). The robot agent is the robot EDDIE (developed at the Technical University of Munich).

$84 \times 62 \mathrm{~mm}(300 \times 300$ DPI $)$ 


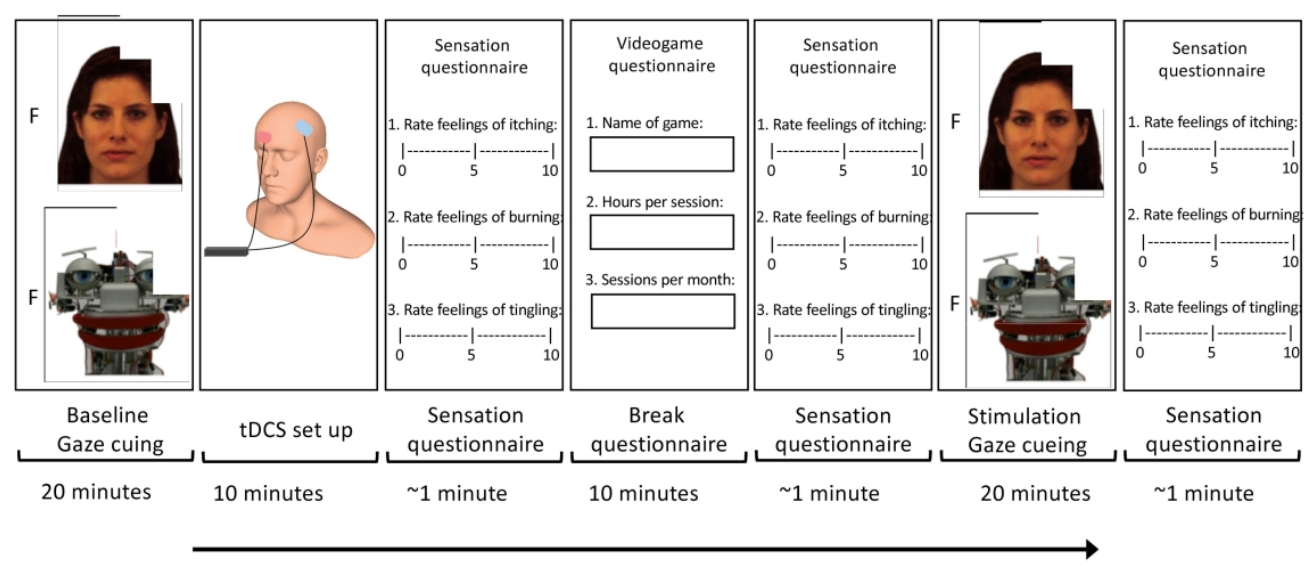

Figure 3. Timing of the Experimental Procedure: The experiment started with participants completing the baseline gaze-cueing task. Next the researcher set up the tDCS machine and participants completed a questionnaire about their sensations. The participants then completed a decoy survey, which asked about their video-game experience. Participants then completed a second sensation questionnaire followed by a gaze-cueing task under stimulation. After the stimulation gaze cueing task was completed, a final sensation questionnaire was administered and the tDCS stimulation was stopped (set-up image was adapted from Dayan et al., 2013).

\section{$270 \times 152 \mathrm{~mm}(300 \times 300 \mathrm{DPI})$}


Figure 4. Sequence of events on a trial of gaze cueing: participants first fixated on a fixation cross for $700-$ $1000 \mathrm{~ms}$ and were then presented with the gazing agent (human vs. robot) looking straight for 700-1000 $\mathrm{ms}$, followed by a change in gaze direction (either to the left or right side of the screen). After an SOA of 400-600 ms, the target letter ( $F$ or $T$ ) appeared either where the face was looking or opposite of where the face was looking. The target remained on the screen until a response was given or a timeout of $1200 \mathrm{~ms}$ was reached.

\section{$279 \times 215 \mathrm{~mm}(300 \times 300$ DPI $)$}




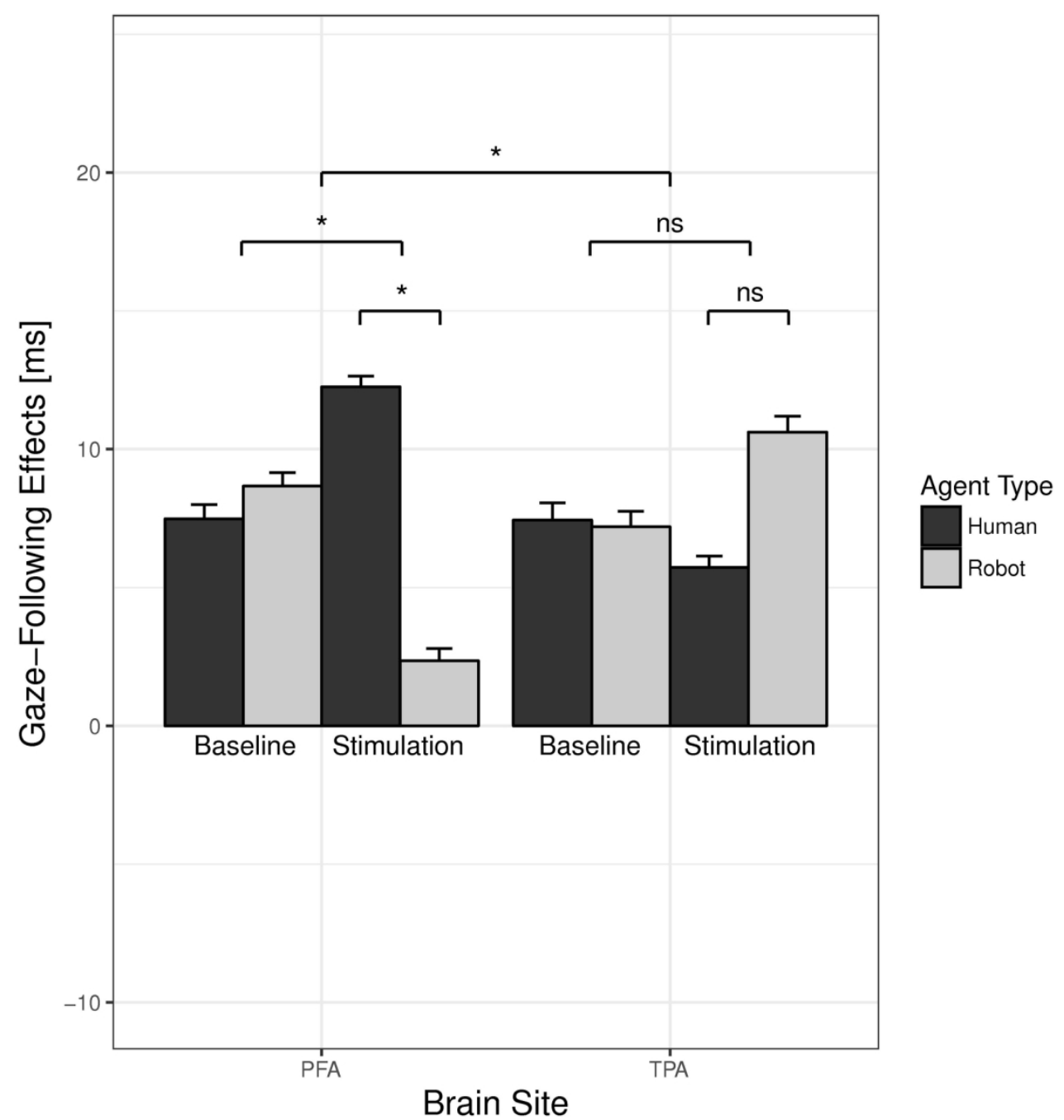

Figure 5. Gaze-cueing effects (in $\mathrm{ms}$ ) as a function of Brain Site (left PF, left TP), Session (Baseline, Stimulation), and Agent Type (Human, Robot). There was a significant change in gaze cueing for active PF stimulation, with no differences in gaze cueing between human and robot at baseline, but significantly larger gaze-cueing effects for the human versus the robot agent under stimulation. Active TP stimulation did not have significant effects on gaze cueing $(* p<.05)$.

$$
156 \times 163 \mathrm{~mm}(300 \times 300 \mathrm{DPI})
$$




\title{
Brain stimulation to left prefrontal cortex modulates attentional orienting to gaze cues
}

\author{
Eva Wiese*, Abdulaziz Abubshait*, Bobby Azarian \& Eric J. Blumberg \\ George Mason University \\ *shared first authorship
}

\section{Corresponding author:}

Eva Wiese

Department of Psychology

Social and Cognitive Interactions Lab

George Mason University

ewiese@gmu.edu 


\section{MEDIA SUMMARY \\ Social robots are increasingly becoming a part of everyday life, and researchers are confronted with the challenge of how to make them sufficiently social for humans to accept them as social companions. Previous research has shown that the effect of nonverbal signals like changes in gaze direction on social-cognitive processes in human-robot interaction depends on the degree to which the robot is perceived as "having a mind". In using brain stimulation, the paper shows that this modulatory effect of mind perception on social-cognitive processes in human-robot interaction likely originates from left prefrontal brain areas.}




\begin{abstract}
In social interactions, we rely on nonverbal cues like gaze direction to understand the behavior of others. How we react to these cues is determined by the degree to which we believe that they originate from an entity with a mind capable of having internal states and showing intentional behavior, a process called mind perception. While prior work has established a set of neural regions linked to mind perception, research has just begun to examine how mind perception affects social-cognitive mechanisms like gaze processing on a neuronal level. In the current experiment, participants performed a social attention task (i.e., attentional orienting to gaze cues) with either a human or a robot agent (i.e., manipulation of mind perception) while transcranial direct current stimulation (tDCS) was applied to prefrontal and temporo-parietal brain areas. The results show that temporo-parietal stimulation did not modulate mechanisms of social attention, neither in response to the human nor the robot agent, whereas prefrontal stimulation enhanced attentional orienting in response to human gaze cues and attenuated attentional orienting in response to robot gaze cues. The findings suggest that mind perception modulates low-level mechanisms of social cognition via prefrontal structures, and that a certain degree of mind perception is essential in order for prefrontal stimulation to affect mechanisms of social attention.
\end{abstract}




\section{INTRODUCTION}

People are seeing a day-to-day increase in the number of robot agents in their lives that could assist them in various domains [1]. Although evidence of positive outcomes of humanrobot interactions exist [2-4], designing social robots that elicit natural human responses can be challenging due to people's negative perceptions about having social robots be a part of their everyday life $[5,6]$. To remedy this and design robots that are able to elicit social responses, we must understand how the human brain processes social information in interactions with others and whether nonhuman agents are able to activate these networks to a similar extent as human interaction partners (and if so, under which conditions). Following this approach, we can identify physical and behavioral agent features that reliably activate brain areas involved in social-cognitive processes and investigate whether robots that elicit activation in these networks lead to more acceptance and trust, as well as improved performance in human-robot interaction [58].

Meaningful social interactions require the ability to infer internal states of others, such as intentions (i.e., mentalizing) and emotions (i.e., empathizing) [7], and to use this information to predict future behavior [8]. For that purpose, the human brain is equipped with neural networks specialized in processing information relevant to social interactions (i.e., social brain; [8-10]) that involve posterior areas like the temporo-parietal junction (TPJ), the superior temporal sulcus (STS) and the fusiform gyrus (FG), as well as anterior areas like the anterior cingulate cortex $(\mathrm{ACC})$ and the ventromedial and dorsolateral prefrontal cortex ( $\mathrm{VmPFC}, \mathrm{dlPFC})$ $[8,11-16]$. TPJ is involved in inferring higher-order action goals [17-25] and mental and spatial perspective taking $[22,24,26]$, while STS and FG are involved in processing biological motion and 
face identity, respectively $[8,15]$. PFC is involved in making inferences about enduring dispositions such as preferences or beliefs $[14,23,27,28]$, and activation in medial PFC is positively correlated with the amount of background knowledge we have about others $[18,29]$. ACC is activated in social interactions requiring mentalizing in real-time [25,30], and is more strongly activated when interacting with human versus machine agents (i.e., computers; [31]). Critically for human-robot interaction, most current social robot platforms underactivate the social brain network $[31,41,44]$, which negatively impacts social (e.g., joint attention), emotional (e.g., empathy) and cognitive (e.g., trust) processes that would be essential in order for humans to socially interact with robot agents. Fortunately, for social roboticists, social brain activation depends on the degree to which an interaction partner is perceived as "having a mind" (i.e., mind perception [32]), with the general capability of making changes in the environment (i.e., agency; [33]), and experiencing internal states, such as emotions and intentions (i.e., experience), and as such can presumably be triggered by design. Whereas mind is easily perceived in other human agents [34], the degree to which nonhuman entities like robots trigger mind perception depends on whether their physical and behavioral characteristics are perceived as sufficiently human-like [35-38]. Mind perception is in fact a highly effortless process that activates social brain networks in a bottom-up or reflexive fashion [133-136], triggered by human-like facial features and relations (i.e., spatial arrangement of eye-nose-mouth configurations) $[131,132,134,135,137]$, as well as biological motion and/or predictable behavior [49].

$\underline{\text { Due to its reflexive nature, mind perception allows observers to differentiate intentional }}$ from non-intentional agents within a few hundred milliseconds [135, 136], and even just 
passively viewing stimuli that trigger mind perception is sufficient in order to induce activation in a wide range of social brain networks [126], which varies parametrically as a function of the agent's physical human-likeness (i.e., increases as the agent's face or body becomes more human-like in appearance) $[57,84]$. Brain areas related to variations in mind perception involve anterior social brain areas, such as the left ACC $[12,25,43]$, as well as posterior areas, such as the left TPJ [90]. Left ACC is activated when others are treated as intentional agents [43, 141], and responds more strongly during social decision making tasks that involve intentional versus non-intentional agents [43, 141]; activation within left TPJ is associated with attributing humanlikeness and intentionality to non-human agents [138-140], and gray matter volume in left TPJ corresponds to individual differences in anthropomorphizing non-human entities, in particular animals [90]. The degree to which agents trigger mind perception not only modulates activation in social brain areas, it also determines how we feel about them [39-42], behave towards them $[31,43-46]$ and interact with them [47-50], and as such has the potential to impact acceptance, trust and performance in human-robot interactions. Mind perception affects higher-order $\underline{\text { social-cognitive processes like prosociality, morality and economic decision-making [46,49], and }}$ also modulates low-level social-cognitive processes, such as face perception [51] and social attention [52-55]. The effect of mind perception on social cognition is so profound that the mere belief that observed behavior may reflect the actions of an agent "with a mind" makes people interpret pre-programmed behaviors as intentional and motivates them to attune their actions accordingly [44,54-56].

Although the neural link between mind perception and social brain activation [27,57], as well as the behavioral link between mind perception and social_cognitive processes are 
relatively well understood [58], research has just begun to examine how mind perception modulates social cognition on a neural level (i.e., activation of which brain areas modulates social cognition as a function of mind perception [ [44]. To address this important issue, we use transcranial direct current stimulation (tDCS) to investigate the link between activation of social brain areas implicated in mind perception and low-level social cognitive processes, such as social attention (i.e., the degree to which changes in gaze direction are followed) [67]. TDCS is a non-invasive electrical stimulation technique that can be applied without disrupting the participant during task execution, and has been proven to be effective in modulating a wide range of cognitive processes in previous studies (e.g., memory, attention, decision-making, perception; [59-62]). Social attention, or the tendency to follow changes in others' gaze direction, was chosen for the present study, as it is a basic, yet essential, social-cognitive mechanism that allows for initiating and coordinating communication [63-65], establishing joint attention between two interaction partners and an object of interest in the environment [66], and an important precursor for developing a theory of mind [66].

Social attention can be examined using a gaze-cueing paradigm, where a face-like stimulus is presented centrally on a screen that first looks straight and then changes gaze direction to the left or right side of the screen. This so-called gaze cue is followed by the presentation of a target stimulus (e.g., F or T) that either appears at the cued location (i.e., valid trial) or an uncued (i.e., invalid trial) location and triggers shifts of the observer's attention to the gazed-at location. As a result, reaction times on valid trials are usually shorter than reaction times on invalid trials, with the difference in reaction times between invalid and valid conditions constituting the gaze-cueing effect [67]. Although it is widely accepted that social 
attention has a strong bottom-up component (i.e., attention is shifted reflexively to the cued location; [66]), there is accumulating evidence that it can be top-down controlled by higherorder social-cognitive processes when context information is available that increases the social relevance of observed gaze signals (e.g., gazer is similar or known to the observer; [54,68-77]). With particular relevance to the current study, manipulating the degree to which a gazer is perceived as an intentional being "with a mind" has been shown to modulate social attention, such that gaze-cueing effects are larger in response to human (i.e. intentional) versus machine (i.e., pre-programmed) gazers $[54,55,78,79]$. That this top-down modulation is specifically related to mind perception is supported by experiments showing that although individuals on the autism spectrum reflexively attend to gaze signals, they do not show the reported enhancement of gaze-cueing effects for human agents but an enhancement in attentional orienting in response to robot gaze cues (potentially due to difficulties with inferring internal states underlying human gaze behavior but an increased interest in machine behavior)[79]. While modulatory effects of mind perception on social attention have been examined behaviorally $[46,48]$, how mind perception modulates lower-level social-cognitive mechanisms $\underline{\text { like gaze cueing at the neuronal level still remains an open question. In particular, it is unclear }}$ whether anterior or posterior parts of the social brain network are more strongly involved in modulating social cognition via mind perception. On the one hand, Oezdem et al. showed that believing that an agent's eye movements are human-controlled (i.e., intentional) as opposed to pre-programmed (i.e.,non-intentional) modulated activation in bilateral TPJ (but not prefrontal networks) ${ }_{2}$ and enhanced attentional orienting to gaze cues [44]. On the other hand, Wiese et al. showed that although variations in social attention in response to human versus robot 
gazers correlated with activation in anterior (i.e., ventromedial PFC) and posterior social brain areas (i.e., TPJ), only activation in bilateral vmPFC correlated with both variations in mind perception and social attention, suggesting that vmPFC might be involved in the top-down control of social attention via mind perception [57]. Taken together, these findings show that although there is convincing evidence that social attention can be modulated via mind perception, the exact source of this modulatory effect still needs to be identified.

To address inconsistencies of previous studies and to examine whether anterior and/or posterior areas of the social brain network_implicated in mind perception are causally involved in modulating social attention, in the current study we compare gaze-cueing effects induced by agents "with a mind" (i.e., human) to agents "without a mind" (i.e., robot), with and without tDCS stimulation to left prefrontal and left temporo-parietal areas. This manipulation was chosen for two reasons: First, previous studies showed that sophisticated minds are attributed to agents with human-like appearance but not to agents with a robot-like appearance (i.e.,

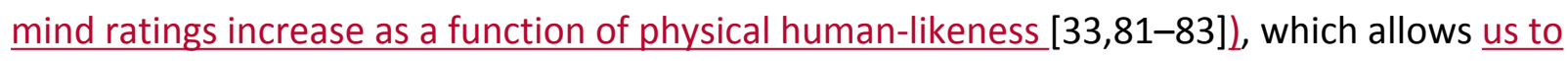
experimentally manipulate mind perception via physical human-likeness. Second, since mind perception increases activation in social brain networks [84-87] and has been shown to

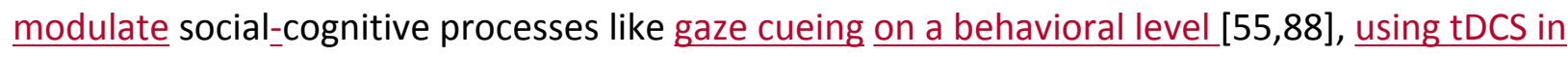
the context of a social attention paradigm seems suitable to investigate the outlined research goal. Left prefrontal and left temporo-parietal areas were chosen as sites for tDCS stimulation for the following reasons: First, both areas have been implicated in mind perception in previous

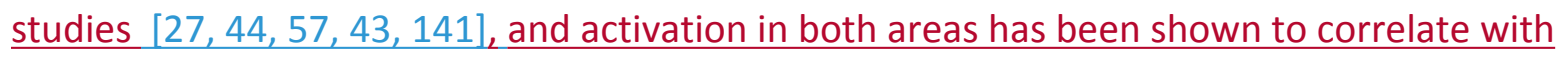
variations in social attention (i.e., gaze cueing) $[44,57]$. Second, both areas are located distant 
enough from each other to minimize the risk of accidental stimulation of the respective other site during stimulation (i.e., prefrontal vs. temporo-parietal). Third, previous studies suggest that active stimulation is superior to sham stimulation as a control, as it controls for side effects of stimulation that are not directly associated to brain functionality like stimulation sensations, and allows for drawing specific inferences regarding the origin of the modulation in terms of location, as permitting by the low spatial resolution of tDCS targeting [92,93].

\section{METHODS AND MATERIALS}

\section{Participants}

Eighty-two undergraduate students from George Mason University (57 females, $M=19.84, S D=$ 2.35 , range: $18-29$ years) participated in the current study for course credit. All participants were right-handed, had normal or corrected-to-normal vision, had no known neurological or psychological deficits, were not taking any medications known to affect the central nervous system at the time of the experiment, and had no history of migraines, seizures, or head injuries. All participants provided written consent to participation and were debriefed at the end of the study. Collection and handling of participant data were in accordance with the IRB guidelines (obtained prior to data collection). Data of participants whose accuracy rate in the gaze cueing task during baseline was below $85 \%$ ( 6 participants), who did not follow the instructions properly ( 2 participants), or did not complete the study (2 participants) were excluded from data analysis ( $13 \%$ of participants in total). The remaining 72 participants were quasi-randomly assigned to the experimental condition, that is: active stimulation of the left prefrontal cortex (PFA; $\mathrm{n}=36,23$ females), or the control condition, that is: stimulation of the 
left temporo-parietal cortex (TP $\underline{A} ; n=36,27$ females). To determine the sample size needed for the study, an a priori power analysis was conducted in G*power for a mixed-effects repeated measures design (i.e., one between factor and one within factor). Since mixed-effects repeated measures designs in G*power can only handle two-factorial designs, the sample size was determined for an ANOVA with a 2-level between factor and a 4-level within factor (i.e., the two within variables were combined). The analysis was based on an alpha of $(\alpha=.05)$, the power set to $(1-\beta=.95)$, and the assumption of a small-to-medium effect size (Cohen's $d=$ .17). The analysis showed that an approximate sample size of 76 participants would be sufficient for both experimental groups. Since the current version of G*power cannot test for a 3-way factorial design but only a 2-way factorial design, another power analysis was conducted using R for the general linear model, which is a more generic test. The analysis was done for a $\underline{2 \times 2 \times 2 \text { factorial model that contains three main effects and their interactions (i.e., three 2-way }}$ interactions and one 3-way interaction) using the same alpha $(\alpha=.05)$, beta $(1-\beta=.95)$, and effect size (Cohen's $d=.17$ ) as the previous analysis. The analysis for the general linear model revealed that the study needed a total of 83 participants.

\author{
Apparatus \\ Stimuli were presented on a Dell 1703FP monitor with the refresh rate set at $85 \mathrm{~Hz}$. RT \\ measures were based on standard keyboard responses. Participants were seated approximately \\ $57 \mathrm{~cm}$ from the monitor, and the experimenter ensured that participants were centered with \\ respect to the monitor. The experiment was controlled by Experiment Builder (SR Research Ltd., \\ Ontario, Canada).
}




\section{TDCS stimulation}

An ActivaDose II, ActivaTek system was used to administer the $2 \mathrm{~mA}$ stimulation. Although there is not a universal and ideal level of current stimulation for any one montage, we selected $2 \mathrm{~mA}$ because it is a commonly used level that is within human safety limits [94]. Electrode placement followed the 10-5 EEG system [95], and tDCS was delivered via two $5 \times 5 \mathrm{~cm}$ saline soaked sponges in rubber housing (resulting in a sponge contact area of $3 \times 3 \mathrm{~cm}$ ). For PFA stimulation, the anode was placed on the scalp over F9 and the cathode was placed over Fz. For TPA stimulation, the anode was placed on the scalp over P5 and the cathode was placed extracephalically on the right $[96,97]$. This montage was chosen to minimize erroneous stimulation of non-targeted cortical areas based on brain modeling (see Brain Modeling; for more details).

\section{Brain Modeling}

Stimulation parameters were modeled using Finite Element Models (FEM) within the HD Explore brain modeling software (Soterix Medical, NY, USA). Brain models were obtained based on conductivities from Datta et al., [98]. The brain models for PFA and TPA stimulation were created for $5 \times 5 \mathrm{~cm}$ sponges (as required by Soterix software). However, since the sponges were reduced to $3 \times 3 \mathrm{~cm}$ due to the usage of sponge housings, we used a 160-channel EEG cap to measure the number of electrodes that were not targeted by the $3 \times 3 \mathrm{~cm}$ sponges in order to correct the initial brain models by reducing the number of electrodes stimulated in the brain modeling software to provide the best approximation of the stimulation intensity at each area. 
PFA stimulation reached multiple prefrontal structures, including the left medial prefrontal cortex (IMPFC), dorsolateral prefrontal cortex (IDLPFC), dorsomedial prefrontal cortex (IDMPFC), ventromedial prefrontal cortex (IVMPFC), and anterior cingulate cortex (IACC): Soterix showed an average peak stimulation intensity of $0.34 \mathrm{~V} / \mathrm{m}$ per $1 \mathrm{~mA}$. For TP $\underline{A}$ stimulation, structures that received stimulation included the left temporo-parietal junction (ITPJ), left superior temporal sulcus (ISTS), left precuneus (IPRC), and left fusiform face area (IFFA): Soterix showed an average peak stimulation intensity of $0.33 \mathrm{~V} / \mathrm{m}$ per $1 \mathrm{~mA}$ (see Figure 1 for brain models and Supplementary Materials for exact stimulation intensities of each structure).

Although multiple models were evaluated, the electrode montages for $\underline{P F A}$ and TP $\underline{A}$ stimulation reported under "tDCS stimulation" demonstrated the greatest peak stimulation intensity over the targeted brain regions, with the least amount of stimulation to non-target brain regions. Specifically, FEM modeling illustrated that using an extracephalic cathodal electrode reduces erroneous stimulation for non-targeted cortical areas in the TPA stimulation condition. It is important to note that although recent evidence suggests that an extracephalic electrode may reduce the magnitude of the stimulation effect when compared to scalp placements [99], this is not the case in the current experiment as FEM models suggest that similar stimulation intensities were achieved for PF $\underline{A}$ and $\underline{\text { TPA }}$ stimulation $(0.34 \mathrm{~V} / \mathrm{m}$ vs 0.33 $\mathrm{V} / \mathrm{m}$ per $1 \mathrm{~mA})$. 


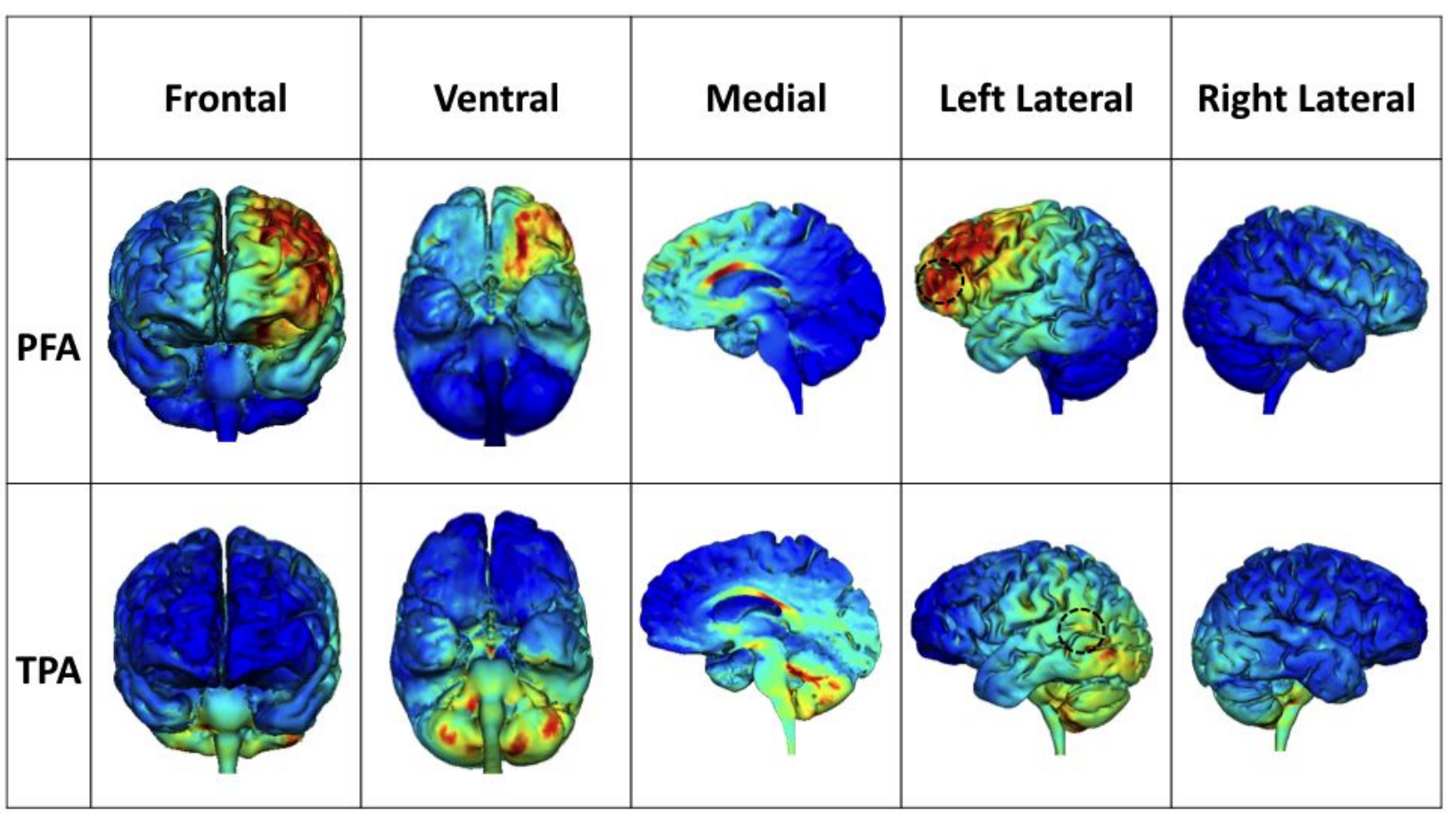

Figure 1. Brain Models illustrate the field intensities of stimulation in $\mathrm{V} / \mathrm{m}$ per $\mathrm{mA}$. Darker red areas illustrate higher stimulation intensities compared to blue areas. The circled regions in the Left Lateral pane show the PFA region and the TPA region, respectively. Please see Figure $\mathbf{1}$ for brain models and Supplementary Materials for exact stimulation intensities of each structure.

\section{Stimuli}

Gaze cueing requires participants to detect, locate or identify targets that are looked-at or looked-away from by a gazer [67], which in the current study is either a human (mind perception high) or a robot (mind perception low). In the human condition, the digitized photo of a female face was used as a gazer, which can be found in the Karolinska Directed Emotional Faces database, while in the robot condition the photo of a humanoid robot was used as gazer (EDDIE; developed by the Technical University of Munich, Germany). The gazing stimuli were $6.4^{\circ}$ wide and $10.0^{\circ}$ high, depicted on a white background and presented in full frontal orientation with eyes positioned on the central horizontal axis of the screen (Figure 2). For left- 
and rightward gaze, irises and pupils of the human and the robot gazer were shifted with Photoshop and deviated $0.4^{\circ}$ from direct gaze. The target stimulus was a black capital letter ( $\mathrm{F}$ or $\mathrm{T}$; measuring $0.8^{\circ}$ in width and $1.3^{\circ}$ in height), which participants had to discriminate by pressing assigned keys on a regular keyboard. The target letters appeared on the horizontal axis of the screen and were located $6.0^{\circ}$ left or right from the center of the screen.

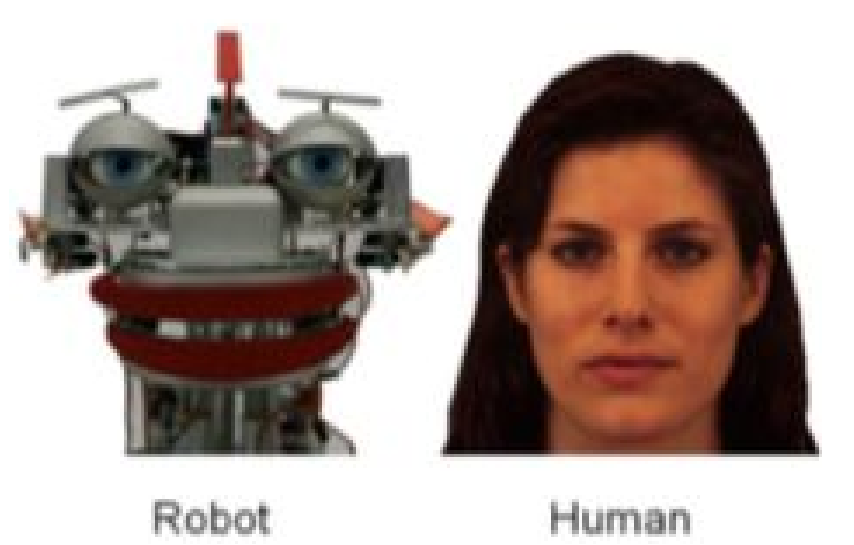

Figure 2. Human and Robot Stimuli: the human agent is represented by a female face taken from the Karolinska Directed Emotional Faces (KDEF) database (F07; written informed consent from Karolinska Institute was received to use the photograph for experimental investigations and illustrations). The robot agent is the robot EDDIE (developed at the Technical University of Munich).

\section{Procedure}

At the beginning of the experiment, participants gave informed consent and completed the Snellen near-sightedness exam to test their eye vision. They then answered a set of questionnaires to assess their perception of robots from the Godspeed measure [100], attitudes towards robots from the Negative Attitude towards Robots questionnaire [101], and autistic traits from the Autism quotient [102]. Upon completion of the questionnaires, participants were instructed how to perform the gaze cueing task, and were informed that the experiment consisted of two parts: a first part where they would perform the gaze cueing task 
without stimulation, and a second part, where they would perform the gaze cueing task under stimulation, with a break for setting up the tDCS equipment in between.

After completing the baseline block, which took 20 minutes, the researcher started setting up the tDCS equipment, which took about 10 minutes. As soon as the current reached its maximum value of $2.0 \mathrm{~mA}$, participants completed a sensation questionnaire to monitor their comfort levels. They were given an unrelated video-game experience questionnaire following the first sensation questionnaire. The unrelated video game questionnaire was administered to ensure that the timing of the stimulation was similar to previous studies that successfully modulated cognitive processes using tDCS $[96,103-106]$. This was an important step as there is no unified standard for the use of tDCS modulation of cognitive tasks [107]. After completing the unrelated videogame questionnaire, participants completed a second sensation questionnaire. Next, subjects started the stimulation gaze-cueing block, which also took 20 minutes. After the stimulation gaze-cueing block, a third sensation questionnaire was administered, the electrodes were removed, the GSM and NARS questionnaire were administered again, and the participants were debriefed. The timing of the experiment can be viewed in Figure 3. 

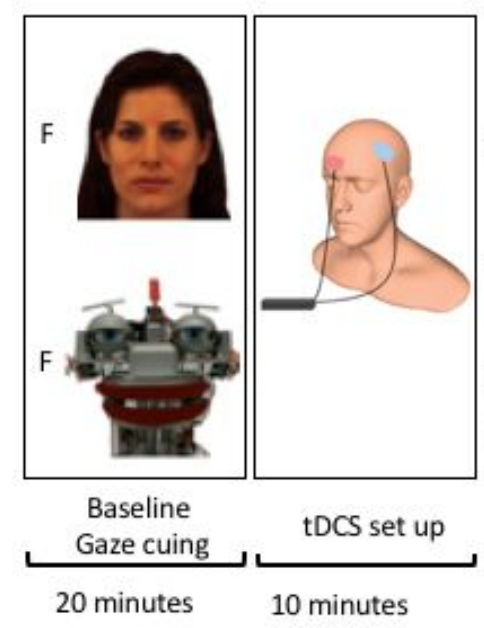

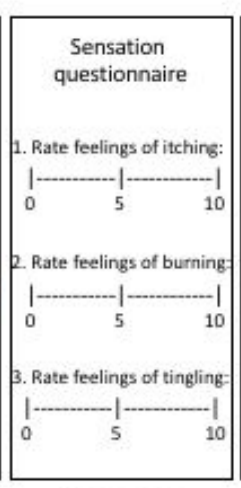

\section{Sensation} questionnaire

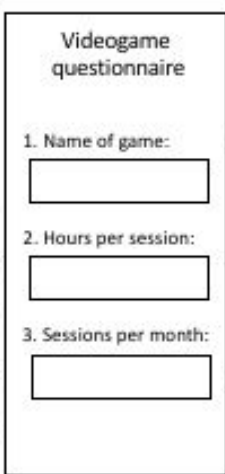

Break questionnaire 10 minutes

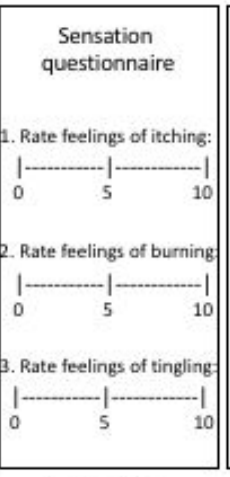

Sensation questionnaire

$\sim 1$ minute

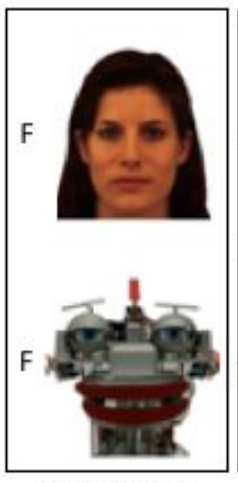

Stimulation Gaze cueing 20 minutes

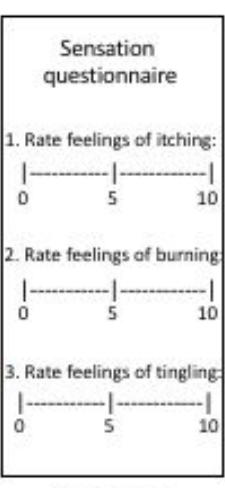

Sensation questionnaire

$\sim 1$ minute

Figure 3. Timing of the Experimental Procedure: The experiment started with participants completing the baseline gaze-cueing task. Next the researcher set up the tDCS machine and participants completed a questionnaire about their sensations. The participants then completed a decoy survey, which asked about their video-game experience. Participants then completed a second sensation questionnaire followed by a gaze-cueing task under stimulation. After the stimulation gaze cueing task was completed, a final sensation questionnaire was administered and the tDCS stimulation was stopped (set-up image was adapted from Dayan et al., 2013).

The sequence of events on a given trial of gaze cueing is illustrated in Figure 4. The beginning of each trial was signaled by a fixation cross at the center of the screen. Between 700-1000 ms later, one of the agents (i.e., human or robot) appeared on the screen looking straight (and with the fixation cross remaining in its position). After a random time interval of 700-1000 ms, the gazer shifted its gaze either left- or rightwards, which constituted the gaze cue. After a stimulus onset asynchrony (SOA; i.e., time interval between gaze cue and target onset) of 400-600 ms, a target letter ( $F$ or T) appeared on the left or the right side of the screen, and participants were asked to respond as fast and accurately as possible to the identity of the 
target [67]. $\stackrel{1}{ }$ Gaze cue and target letter remained on the screen until a response was given or after a timeout of 1200 ms was reached, whichever came first. The next trial started after an inter-trial-interval (ITI) of $680 \mathrm{~ms}$.

Participants were instructed to fix their gaze on the fixation cross at the beginning of a trial, and to not make any eye movements during the trial. They were also instructed that after the fixation cross the image of a social agent would appear in the center of the screen, which would first look at them (mutual gaze), and then after some time make an eye movement to look to the left or right side of the screen (averted gaze). Participants were further advised that the change in gaze direction would be followed by the appearance of a target letter ( $F$ or T), which would appear either at the gazed-at location or opposite of the gazed-at location. Participants were asked to indicate as quickly and accurately as possible whether " $\mathrm{F}$ " or " $\mathrm{T}$ " was shown on the screen by pressing the respective response key: For one half of the participants " $\mathrm{F}$ " was assigned to the " $\mathrm{D}$ " key and " $\mathrm{T}$ " to the " $\mathrm{K}$ " key on a regular keyboard, while for the other half of participants stimulus-response mapping was reversed. The original key labels were covered with a sticker to prevent interference effects with the actual letters on the keyboard. All instructions were given in written form.

Gaze direction (left, right), target location (left, right), target identity $(F, T)$ and agent type (human, robot) were selected pseudo-randomly; every combination appeared with equal frequency throughout the experiment. Gaze direction was manipulated orthogonal to target

\footnotetext{
${ }^{1}$ The SOAs were jittered to prevent preparedness effects on the participant's side. Please note that this is in line with previous studies (e.g., [55]) and should not affect attentional orienting, since no qualitative differences in attentional orienting have been reported in this time frame (i.e., both reflexive and voluntary shifts of attention occur in the 400-600 ms SOA range, and not inhibition of return effects have been reported) [66].
} 
location: in half of the trials, the target was validly cued, and in the other half of the trials, the target was invalidly cued. Each experimental session was composed of 220 trials, with a block of 20 practice trials preceding two gaze-cueing blocks of 100 trials of gaze cueing each (i.e., 220 trials for the stimulation baseline and 220 trials for the stimulation block). Participants first completed one block of gaze cueing without stimulation, and were then assigned to either the group that received active stimulation to left PFA or the group that received active stimulation to left TPA. Stimulation was applied for 30 minutes, during which participants completed the second block of gaze cueing.

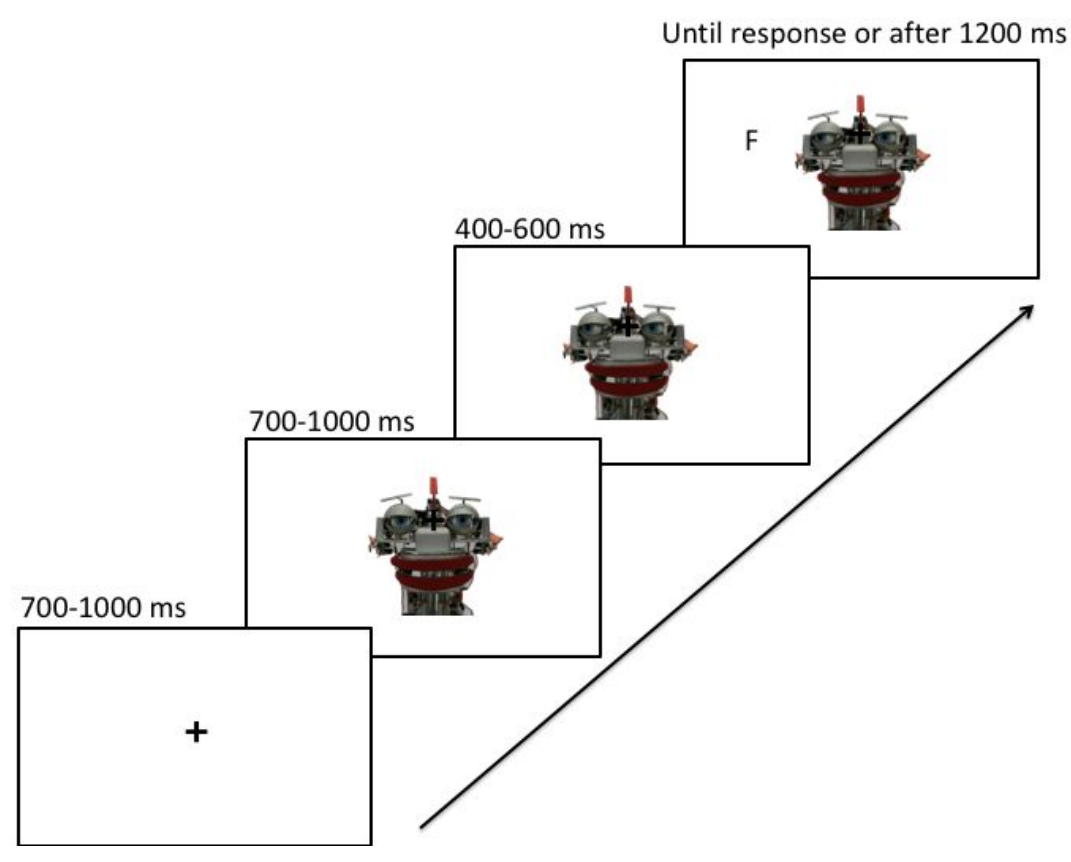

Figure 4. Sequence of events on a trial of gaze cueing: participants first fixated on a fixation cross for 700-1000 ms and were then presented with the gazing agent (human vs. robot) looking straight for 700$1000 \mathrm{~ms}$, followed by a change in gaze direction (either to the left or right side of the screen). After an SOA of 400-600 ms, the target letter (F or T) appeared either where the face was looking or opposite of where the face was looking. The target remained on the screen until a response was given or a timeout of 1200 ms was reached. 


\section{RESULTS \\ Questionnaires}

We used the Godspeed Measure (GSM; [100]) and the Negative Attitude towards Robots Scale (NARS; [101]) to determine whether participants in the two different stimulation conditions differed in their perception of and attitudes towards robots. Both questionnaires were administered at the beginning of the experiment (to capture potential a-priori individual differences), as well as after the completion of the experiment (to assess whether attitudes changed). The short version of the Autism Quotient (AQ-short; [102]) was administered once, at the beginning of the experiment, to measure participants' autistic traits. A 10-point sensation questionnaire was also administered three times to monitor participant's comfort levels during the experiment $[96,97]$. Participants were told that a reporting a "7" would illustrate unbearable sensations. The questionnaire was administered as soon as stimulation started, before the start of the stimulation block, and after completing the stimulation block. Participants in the PFA and the TPA stimulation did not differ in terms of their perception of robots $\left(G S M ; F(1,69)=.09, p=.75 ; \eta^{2}<.001\right)$, attitudes towards robots (NARS; $F(1,69)=.2$, $\left.p=.65 ; \eta^{2}<.01\right)$ or autistic traits (AQ score; $\left.t(69)=-1.66, p=.09\right)$. Comparison of the GSM and NARS pre-post ratings did not reveal differences between the two stimulation conditions (GSM: $F(1,69)=1.19, p=.27, \eta^{2}<.01 ;$ NARS: $\left.F(1,69)=.04, p=.84, \eta^{2}<.001\right) ;$ see Tables S1 and S2 in the Supplementary Materials for the full report of the inference statistics. Two participants indicated sensation levels of above "7", which indicated that they were uncomfortable and did not continue the experiment (see participants section). 


\section{Behavioral Data}

To determine whether active PFA and/or TPA stimulation had a modulatory effect on gaze cueing, we conducted a $2 \times 2 \times 2$ mixed ANOVA on gaze cueing effects (i.e., mean reaction times for invalid - valid trials) with Agent Type (human vs. robot) and Session (baseline vs. stimulation) as within-participants factors, and Brain Site (left PFA vs. TPA) as between-participants factor. The descriptive statistics of this analysis are depicted in Figure $\mathbf{5}$.

Before examining the results of the statistical models, we tested the normality of the residuals using the Kolmogorov-Smirnov test (and not the more commonly used Shapiro-Wilk test as is it not recommended for larger sample sizes; $[108,109])$. The Kolmogorov-Smirnov test revealed a non-significant effect $(D=.07, p=.42)$, showing that the residuals of our data did not differ significantly from a normal distribution (i.e., the normality of the residuals assumption of parametric tests was not violated).

The ANOVA revealed no main effect of Agent Type $\left(\mathrm{F}(1,70)=.2, \mathrm{p}=.64, \mathrm{n}^{2}=<.001\right)$, indicating that across stimulation sites and sessions, there were no significant differences in gaze-cueing effects for the human agent compared to the robot agent (Human: 8.22 ms vs. Robot: $7.20 \mathrm{~ms})$. The main effect of Session $\left(F(1,70)<.001, p=.98, \eta^{2}<.001\right)$ was also not significant, showing that across stimulation sites and agent types gaze cueing effects did not differ between baseline and stimulation (Baseline: 7.69 ms vs. Stimulation: $7.74 \mathrm{~ms}$ ). The main effect of Brain Site $\left(F(1,70)<.001, p=.98, \eta^{2}<.001\right)$ was also not significant, showing that across agent types and sessions, no differences in gaze cueing effects were found between the two stimulation sites (PFA: $7.69 \mathrm{~ms}$ vs. TPA: $7.74 \mathrm{~ms}$ ). All two-way interactions were also not significant (Agent Type $\times$ Session: $F(1,70)=.61, p=.43, \eta^{2}<.001 ;$ Agent Type $\times$ Brain Site: $(F(1$, 
$\left.70)=2.24, p=.13, \eta^{2}<.01\right) ;$ Session $\times$ Brain Site: $\left(F(1,70)=.17, p=.67, \eta^{2}<.001\right)$. Most importantly, however, the three-way interaction of Agent Type $\times$ Session x Brain Site was significant $\left(F(1,70)=4.50, p=.03, \eta^{2}=.12\right)$ indicating that tDCS stimulation affected gaze cueing effects differently for the human versus the robot condition under left PFA but not left TPA stimulation.

To examine the significant three-way interaction effect further, two $2 \times 2$ post-hoc ANOVAs with Agent Type (Human vs. Robot) and Session (Baseline vs. Stimulation) were conducted, one for left PFA stimulation and one for left TPA stimulation. The ANOVA for the PFA stimulation condition showed no main effects of Agent Type $(F(1,35)=2.21, p=.14$, $\left.\eta^{2}=.01\right)$ or Session $\left(F(1,35)=.08, p=.77, \eta^{2}<.001\right)$, but a significant Agent Type $\times$ Session interaction $\left(F(1,35)=5.51, p=.02, \eta^{2}=.02\right)$. Post-hoc paired t-tests revealed that there was no significant difference in gaze cueing effects between the human and robot agents at baseline (Human: 7.47 ms vs. Robot: $8.66 \mathrm{~ms} ; \mathrm{p}=.75$ ), but significantly larger cueing effects for the human versus the robot gazer under stimulation (Human: 12.25 ms vs. Robot: $2.34 \mathrm{~ms} ; \mathrm{p}=$ .02). In contrast, the ANOVA for the TPA stimulation condition revealed neither main effects of Agent Type $\left(\mathrm{F}(1,35)=.47, \mathrm{p}=.49, \mathrm{\eta}^{2}<.01\right)$ or Session $\left(\mathrm{F}(1,35)=.09, \mathrm{p}=.76, \eta^{2}<.01\right)$, nor a significant Agent Type $x$ Session interaction $\left(F(1,35)=.72, p=.39, \eta^{2}<.01\right)$. This suggests that while PFA stimulation modulated gaze cueing effects with significantly larger gaze-cueing effects for the human versus the robot agent under stimulation, TPA stimulation did not have such a modulatory effect on gaze cueing (i.e., no differences in gaze cueing at baseline and under stimulation). Post-hoc t-tests were corrected using the false discovery rate procedure (FDR). 
A separate $2 \times 2 \times 2$ mixed ANOVA was conducted on accuracy ratings of participants.

The ANOVA revealed no significant main effects or interaction effects. See the Behavioral

Results section of the Supplementary Materials for inference statistics. All data and materials can be publicly viewed on https://osf.io/s8ewg/.

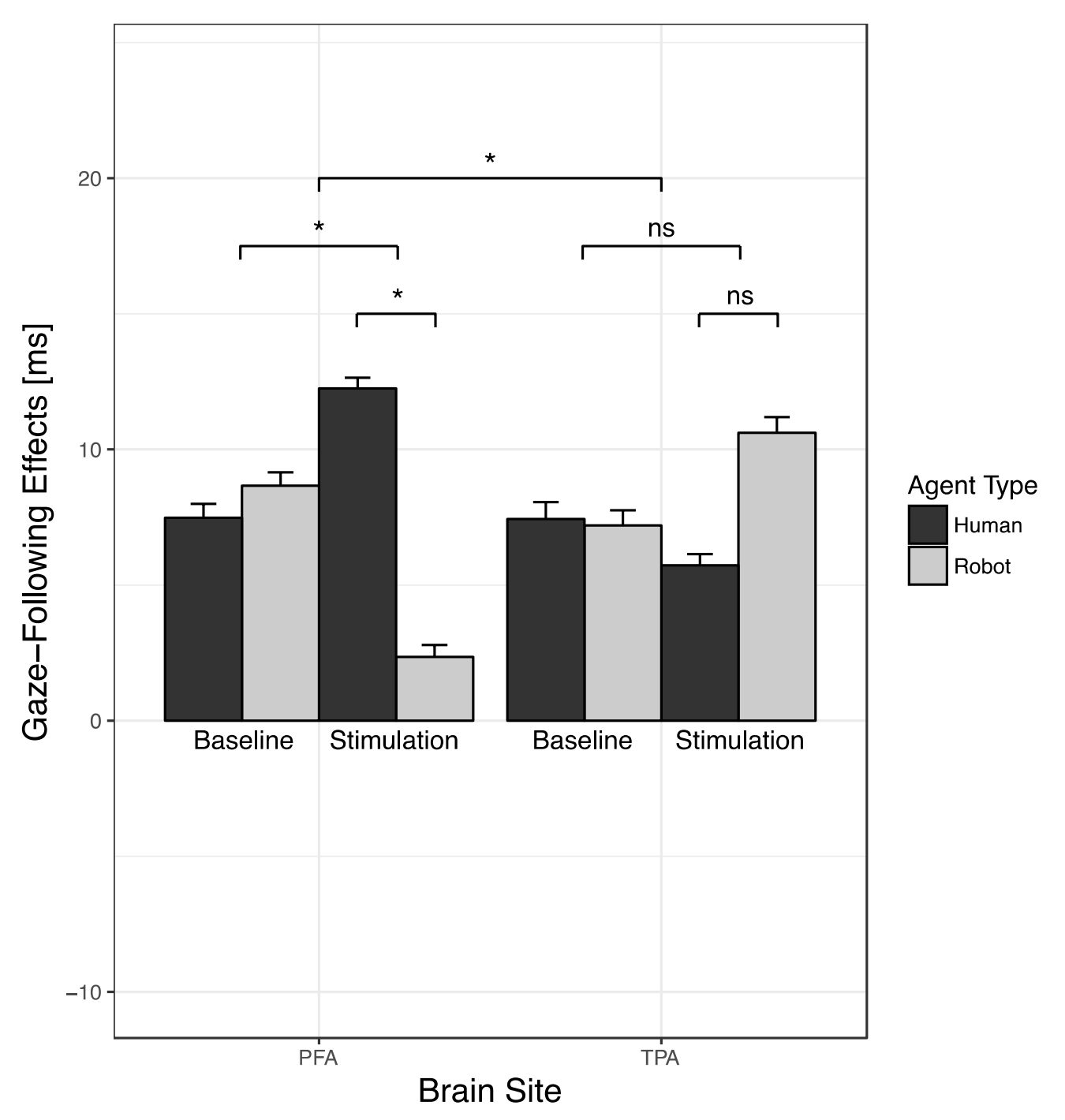

Figure 5. Gaze-cueing effects (in ms) as a function of Brain Site (left PFA, left TPA), Session (Baseline, Stimulation), and Agent Type (Human, Robot). There was a significant change in gaze cueing for active PFA stimulation, with no differences in gaze cueing between human and robot at baseline, but significantly larger gaze-cueing effects for the human versus the robot agent under stimulation. Active TPA stimulation did not have significant effects on gaze cueing $(* p<.05)$. 


\section{DISCUSSION}

Previous studies have shown that activation in left prefrontal $[10,31,43,57,110,111]$ and temporo-parietal $[25,27,43,90]$ areas is related to mind perception and the modulation of lowlevel social-cognitive processes like gaze cueing $[44,57,58]$. The goal of the current experiment was to examine the causal involvement of left prefrontal and left temporo-parietal areas in the top-down modulation of social attention via mind perception. To address this issue, we asked participants to perform a gaze-cueing task with a human and a robot agent (i.e., manipulation of mind perception via physical human-likeness) while applying tDCS to left prefrontal and left temporo-parietal areas. The findings show that stimulating left prefrontal had a modulatory effect on social attention, such that gaze cues of intentional agents (i.e., human) were followed significantly more strongly than gaze cues of machine agents (i.e., robot) under stimulation of prefrontal areas. Left temporo-parietal stimulation, in contrast, did not significantly modulate gaze-cueing effects for either of the two agents. These results are in line with previous studies showing that experimental manipulations of mind perception enhance the degree to which gaze signals are followed $[44,54,55]$. In particular, it was shown that interpreting gaze signals as intentional or human-controlled augments sensory processing of stimuli presented at gazed-at locations [54], and increases the social relevance of observed gaze signals $[53,55,79,112]$. The results are also in line with studies showing a correlational relationship between activation in left prefrontal areas related to mind perception and modulation of social attention when performing an orthogonal gaze cueing task outside the fMRI scanner [57], as well as tDCS studies showing that temporo-parietal areas are causally involved in social-cognitive processes 


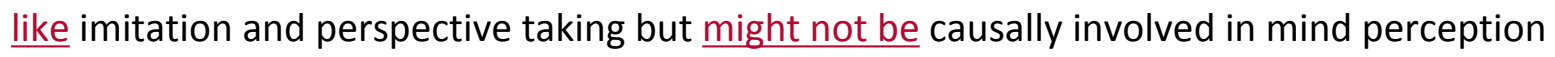
[113-115].

The current experiment adds to previous findings by localizing the source of top-down modulation of social attention via mind perception to left prefrontal areas, including areas like

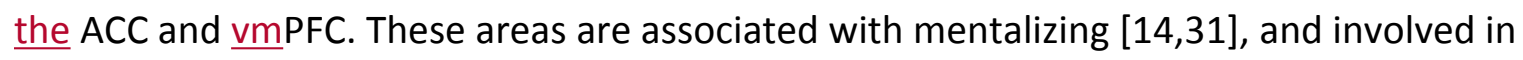
impression formation in social interaction $[116,117]$. In particular, activation in MPFC is linked to retrieving stereotypical knowledge about other people [118-120], and associated with retrieving script-based social knowledge [121,122]. Medial prefrontal areas are also more strongly activated when mentalizing about the internal states of similar than dissimilar others $[117,123-125]$, as well as when viewing social scenes that contain human versus nonhuman agents [126]. The current experiment adds to the literature by indicating that prefrontal areas might not only is involved in the modulation of higher-order social-cognitive processes like decision-making $[31,43]$, but might also exert modulatory effects on low-level social cognitive processes like social attention.

Please note that the current experiment does not show the previously reported difference in gaze cueing between human and robot gazer at baseline [55]. This can be due to several reasons: First, in previous experiments, the gazers were introduced as "human" versus "robot" via instruction, which provided participants with explicit labels as to how to treat them in terms of mind perception (i.e., "human/has a mind" and "robot/has no mind"). In contrast, in the current experiment, the gazers were introduced more neutrally as "agents" and their mind status had to be inferred from physical appearance, which makes the mind perception manipulation more implicit. Although this certainly increases the external validity of the 
experimental manipulation, it is possible that participants did not pay enough attention to the gazers' mind status, which could wash out effects between the two agents at baseline. Second, since mind perception was manipulated via physical appearance in the current experiment, it is also possible that individual differences in anthropomorphism $[89,90]$ attenuated differences in gaze cueing between the human and robot gazer at baseline. However, since baseline effects are comparable for both stimulation conditions and since the current paper is mainly interested in the modulation of social attention via mind perception, insignificant differences in gaze cueing at baseline should not have impacted the reported findings. Nevertheless, in order to validate the robustness of the reported findings, future experiments should be conducted to determine to which degree they might be influenced by individual differences in anthropomorphism.

The question remains why stimulation of left temporo-parietal networks did not significantly modulate low-level mechanisms of social cognition despite previous reports showing a correlational relationship between mechanisms of social attention and bilateral TPJ activation [44]. One explanation for the lack of a significant effect of left temporo-parietal stimulation on gaze cueing is that it is possible that processes related to mind perception and social attention are not sufficiently interconnected at the level of the left TPJ in order to exert a top-down modulatory effect on attentional orienting to gaze cues. This interpretation would be in line with previous reports showing that social functions within the TPJ are lateralized [17], and that an overlap between attentional orienting and mentalizing is found within the right but not left TPJ $[11,127,128]$. In contrast, left TPJ lesions have been shown to cause selective deficits in false belief reasoning $[89,91]$, which does require mentalizing but no orientation of 
social attention. In support of this notion, it has been shown that early posterior ERP components like the N170 are sensitive to the intentionality of an agent without being responsive to the congruency or social outcome of its gaze cues [112], whereas later anterior ERP components like the P350 are sensitive to both an agent's intentionality and the congruency of gaze cues [78] (i.e., significant difference in P350 amplitudes for invalid vs. valid trials for human vs. computer-controlled conditions), suggesting that the integration of mind perception related processes and social attention might be instantiated in prefrontal (but not temporo-parietal) areas.

Another possible explanation is that prefrontal and temporo-parietal brain regions might process information about an agent's mind on different levels, with TPJ activation being related to inferring particular internal states from observed behaviors $[11,19,27,110]$ (e.g., observing an agent smile leads to the inference that the agent is currently in a state of happiness), and MPFC and VMPFC activation being related to reasoning based on stereotypical assumptions regarding general traits associated with intentional agents [129] (e.g., agent that looks like a child might like toys). It would be possible that studies that manipulate mind perception via instruction of particular beliefs (i.e., "eye movements are intentional") engage the posterior mind perception network [44], while studies that manipulate mind perception via physical appearance (i.e., the agent looks human) more strongly activate stereotypical assumptions about human behavior, thereby engaging the anterior mind perception network involving the MPFC and VMPFC [118-120]. This interpretation is particularly plausible given that attentional orienting to gaze cues is a fast-acting process [67], which requires information from a readily available source in order to be top-down controlled. Since stereotypical 
information about an agent is more readily available than the outcomes of mentalizing processes about particular internal states, a stronger involvement of prefrontal areas in the top-down modulation of fast processes like social attention seems tenable.

A third explanation is that the observed modulation of gaze cueing is not specific to mind perception, but due to other (related) functions associated with the left prefrontal cortex. One aspect of the experimental design that could have affected social attention in addition to the gazer's physical appearance is the non-predictivity of gaze cues in the current experiment (i.e., targets appear with equal frequency at validly and invalidly cued locations). Previous studies have shown that stimuli whose behavior is hard to predict are more likely anthropomorphized, and that evaluating unpredictable stimuli is associated with increased activation in medial prefrontal areas, and specifically the VMPFC and ACC [49]. In consequence, it is possible that one's sensitivity to the predictability of gaze cues had an impact on the degree to which the gazer was anthropomorphized and prefrontal brain areas were activated during gaze cueing. For the current experiment, this means that stimulating prefrontal areas may have specifically enhanced the social relevance of human gaze cues, leading to longer processing times on invalid trials [78] and larger gaze-cueing effects (i.e., difference in reaction times between invalid and valid trials), while temporo-parietal stimulation may not have affected the perceived social relevance of human gaze cues² .

Alternatively, the VMPFC has been shown to track feelings of eeriness towards nonhuman agents in a parametric fashion [129] and has been labeled the potential neural

\footnotetext{
${ }^{2}$ Please note that the conducted a-priori power analysis was based on a small-to-medium effect size, which leaves the possibility that very small-to-small effects might not have been detected with the current sample size.
} 
correlate of the uncanny valley [130] (i.e., nonhuman agents with human-like appearance induce feelings of eeriness if they are not perfectly human). If that were the case, stimulation of prefrontal areas could have enhanced feelings of eeriness towards the robotic agent, leading to a disengagement from robot gaze cues together with an increased engagement in attending to human gaze cues (i.e., eeriness of robot cues made human gaze cues more "desirable"). Effects related to non-social prefrontal functions such as working memory, executive functioning or abstract reasoning are less likely to have influenced gaze cueing, since one would expect comparable effects of stimulation for human and nonhuman agents. Whether prefrontal stimulation modulated gaze cueing directly via mind perception or via processes affected by mind perception, such as perception of uncertainty [78] or emotional reactions to uncanny agents [129], cannot ultimately be answered based on the current data and requires follow-up studies. It can also not be clearly determined - due to the lack of spatial specificity of tDCS which prefrontal brain area(s) ultimately caused the observed top-down modulation of social attention (i.e., areas directly implicated in mind perception such as the left ACC, or areas that are indirectly involved in mind perception such as the left MPFC, VMPFC or DLPFC).

\section{CONCLUSIONS}

Previous studies have shown that the degree to which we attend to social cues depends on the degree to which we perceive mind in the entity sending the cues. The neural correlates of mind perception have been localized to prefrontal and temporo-parietal structures in previous studies, but the causal involvement of these areas in the modulation of low-level socialcognitive processes like gaze cueing has not been determined yet. The current study shows that 
stimulation to prefrontal areas increases the degree to which human gaze is followed compared to the degree to which robot gaze is followed, while stimulation to temporo-parietal regions does not seem to have a measurable modulatory effect on gaze cueing. Since the effect of prefrontal stimulation is only observable for human gazers, it is tenable that prefrontal stimulation does not simply lead someone to perceive "more" mind in others, but rather seems to enhance the social relevance of signals coming from agents "with a mind". In other words, prefrontal stimulation does not seem to make participants perceive more human-likeness in non-human agents, which makes it unlikely that the observed effect is related to anthropomorphism. Instead, prefrontal stimulation seems to help discriminate agents "with a mind" from agents "without a mind", as evidenced by an increased difference in gaze cueing between the human and the robot gazer under stimulation, indicating that stimulation of prefrontal areas enhances the importance of social signals coming from human agents. Taken together, this study shows a causal link between prefrontal stimulation and mechanisms of social attention, and dissociation between the anterior and posterior part of the social brain network in terms of top-down modulation of social-cognitive processes. Whether the effect is specific to mind perception or related to processes indirectly affected by mind perception needs to be determined in future studies.

\section{REFERENCES}

1. Tapus A, Matarić M. Towards socially assistive robots. J Robot Soc Jpn. 2006; 14(5):5768.

2. Scassellati B, Admoni H, Matarić M. Robots for Use in Autism Research. Annu Rev Biomed Eng. 2012;14:275-94.

3. Basteris A, Nijenhuis SM, Stienen AH, Buurke JH, Prange GB, Amirabdollahian F. Training modalities in robot-mediated upper limb rehabilitation in stroke: a framework for classification based on a systematic review. J NeuroEngineering Rehabil. 2014;11(1):111. 
4. Mubin O, Stevens CJ, Shahid S, Mahmud AA, Dong J-J. A REVIEW OF THE APPLICABILITY OF ROBOTS IN EDUCATION. Technol Educ Learn [Internet]. 2013 [cited 2018 Nov 5];1(1). Available from: http://www.actapress.com/PaperInfo.aspx?paperld=43268

5. Bartneck C, Reichenbach J. Subtle emotional expressions of synthetic characters. Int J Hum-Comput Stud. 2005 Feb;62(2):179-92.

1.6. Scopelliti M, Giuliani MV, Fornara F. Robots in a domestic setting: a psychological approach. Univers Access Inf Soc. 2005 Dec;4(2):146-55.

2.7. Baron-Cohen S. Mindblindness: An essay on autism and theory of mind. MIT press; 1997.

3.8. Frith CD, Frith U. How we predict what other people are going to do. Brain Res. 2006;1079:36-46.

4.9. Adolphs R. The Social Brain: Neural Basis of Social Knowledge. Annu Rev Psychol. 2009;60(1):693-716.

5.10. Van Overwalle F, Baetens K. Understanding others' actions and goals by mirror and mentalizing systems: A meta-analysis. Neurolmage. 2009;48(3):564-84.

6.11. Bzdok D, Langner R, Schilbach L, Engemann DA, Laird AR, Fox PT, et al. Segregation of the human medial prefrontal cortex in social cognition. Front Hum Neurosci [Internet]. 2013;7. Available from: http://journal.frontiersin.org/article/10.3389/fnhum.2013.00232/abstract

7.12. Spunt RP, Adolphs R. Validating the Why/How contrast for functional MRI studies of Theory of Mind. Neurolmage. 2014;99:301-11.

8.13. Spunt RP, Lieberman MD. Dissociating Modality-Specific and Supramodal Neural Systems for Action Understanding. J Neurosci. 2012;32(10):3575-83.

9.14. Amodio DM, Frith CD. Meeting of minds: the medial frontal cortex and social cognition. Nat Rev Neurosci. 2006 Apr;7(4):268-77.

10.15. Saxe R, Carey S, Kanwisher N. Understanding other minds: linking developmental psychology and functional neuroimaging. Annu Rev Psychol. 2004;55:87-124.

11.16. Gallagher HL, Frith CD. Functional imaging of 'theory of mind.' Trends Cogn Sci. 2003;7(2):77-83.

12.17. Perner J, Aichhorn M, Kronbichler M, Staffen W, Ladurner G. Thinking of mental and other representations: The roles of left and right temporo-parietal junction. Soc Neurosci. 2006;1(3-4):245-58.

13.18. Grèzes J, Berthoz S, Passingham RE. Amygdala activation when one is the target of deceit: Did he lie to you or to someone else? Neurolmage. 2006;30(2):601-8.

14.19. Saxe R, Powell L. It's the thought that counts: Specific brain regions for one component of theory of mind. Psychol Sci. 2006;17(8):692-9.

15.20. Ohnishi T, Moriguchi Y, Matsuda H, Mori T, Hirakata M, Imabayashi E, et al. The neural network for the mirror system and mentalizing in normally developed children: An fMRI study. NeuroReport. 2004;15(9):1483-7.

16.21. Grezes J. Brain Mechanisms for Inferring Deceit in the Actions of Others. J Neurosci. 2004;24(24):5500-5.

17.22. Farrer C, Franck N, Georgieff N, Frith CD, Decety J, Jeannerod M. Modulating the experience of agency: A positron emission tomography study. Neurolmage. 2003;18(2):324-33. 
18.23. Saxe R, Kanwisher N. People thinking about thinking people: The role of the temporo-parietal junction in "theory of mind." Neurolmage. 2003;1835-42.

19.24. Chaminade T, Decety J. Leader or follower? Involvement of the inferior parietal lobule in agency. Neuroreport. 2002;13(15):1975-8.

20.25. Gallagher HL, Happé F, Brunswick N, Fletcher PC, Frith U, Frith CD. Reading the mind in cartoons and stories: An fMRI study of "theory of mind" in verbal and nonverbal tasks. Neuropsychologia. 2000;38(1):11-21.

21.26. Ruby $P$, Decety J. Effect of subjective perspective taking during simulation of action: a PET investigation of agency. Nat Neurosci. 2001;4(5):546-50.

22.27. Van Overwalle F. Social cognition and the brain: A meta-analysis. Hum Brain Mapp. 2009;30(3):829-58.

23.28. Saxe R. Uniquely human social cognition. Curr Opin Neurobiol. 2006;16(2):235-9. 24.29. Saxe R, Wexler A. Making sense of another mind: The role of the right temporoparietal junction. Neuropsychologia. 2005;43(10):1391-9.

25.30. McCabe K, Houser D, Ryan L, Smith V, Trouard T. A functional imaging study of cooperation in two-person reciprocal exchange. Proc Natl Acad Sci. 2001;98(20):118325.

26.31. Gallagher $\mathrm{HL}$, Jack Al, Roepstorff A, Frith CD. Imaging the intentional stance in a competitive game. Neurolmage. 2002;16:814-21.

27.32. Spunt RP, Meyer ML, Lieberman MD. The Default Mode of Human Brain Function Primes the Intentional Stance. J Cogn Neurosci. 2015 Jun;27(6):1116-24.

28.33. Gray HM, Gray K, Wegner DM. Dimensions of Mind Perception. Science. 2007;315(5812):619-619.

29.34. Epley N, Waytz A, Cacioppo JT. On Seeing Human: A Three-Factor Theory of Anthropomorphism. Psychol Rev. 2007;114(4):864-86.

30.35. Kiesler S, Powers A, Fussell SR, Torrey C. Anthropomorphic interactions with a robot and robot-like agent. Soc Cogn. 2008;26(2):169-181.

31.36. DiSalvo C, Gemperle F. From Seduction to Fulfillment: The Use of Anthropomorphic Form in Design. Int Conf Des Pleasurable Prod Interfaces. 2003;67-72. 32.37. Castelli F, Happé F, Frith U, Frith C. Movement and Mind: A Functional Imaging Study of Perception and Interpretation of Complex Intentional Movement Patterns. Neurolmage. 2000 Sep;12(3):314-25.

33.38. Heider F, Simmel M. An Experimental Study of Apparent Behavior. Am J Psychol. 1944;57(2):243-59.

34.39. Gutsell JN, Inzlicht M. Intergroup differences in the sharing of emotive states: Neural evidence of an empathy gap. Soc Cogn Affect Neurosci. 2012;7(5):596-603.

35.40. Saygin AP, Chaminade T, Ishiguro H, Driver J, Frith $C$. The thing that should not be: predictive coding and the uncanny valley in perceiving human and humanoid robot actions. SCAN. 2012;7:413-22.

36.41. Harris LT, Fiske ST. Perceiving Humanity or Not: A Social Neuroscience Approach to Dehumanized Perception. In: Social Neuroscience: Toward Understanding the Underpinnings of the Social Mind. 2011. 
37.42. Cehajic S, Brown R, Gonzalez R. What do i care? Perceived ingroup responsibility and dehumanization as predictors of empathy felt for the victim group. Group Process Intergroup Relat. 2009;12(6):715-29.

38.43. Sanfey AG, Rilling JK, Aronson JA, Nystrom LE, Cohen JD. The neural basis of economic decision-making in the Ultimatum Game. Science. 2003;300(5626):1755-8.

39.44. Özdem C, Wiese E, Wykowska A, Müller H, Brass M, Van Overwalle F. Believing androids - $\mathrm{fMRI}$ activation in the right temporo-parietal junction is modulated by ascribing intentions to non-human agents. Soc Neurosci. 2016;12(5):582-93.

40.45. Haley KJ, Fessler DMT. Nobody's watching? Subtle cues affect generosity an anonymous economic game. Evol Hum Behav. 2005;26(3):245-56.

41.46. Bering JM, Johnson D. " O Lord... You Perceive my Thoughts from Afar":

Recursiveness and the evolution of supernatural agency. J Cogn Cult. 2005;5(1):118-42. 42.47. Hertz N, Wiese E. Social Facilitation with Non-Human Agents: Possible or not? Proc Hum Factors Ergon Soc Annu Meet. 2017 Sep;61(1):222-5.

43.48. Riether N, Hegel F, Wrede B, Horstmann G. Social facilitation with social robots? In: Proceedings of the seventh annual ACM/IEEE international conference on HumanRobot Interaction - HRI '12 [Internet]. Boston, Massachusetts, USA: ACM Press; 2012 [cited 2018 Aug 15]. p. 41. Available from:

http://dl.acm.org/citation.cfm?doid=2157689.2157697

44.49. Waytz A, Gray K, Epley N, Wegner DM. Causes and consequences of mind perception. Trends Cogn Sci. 2010 Aug;14(8):383-8.

45.50. Short E, Hart J, Vu M, Scassellati B. No fair!! An interaction with a cheating robot. 2010 5th ACMIEEE Int Conf Hum-Robot Interact HRI. 2010;219-26.

46.51. Takahashi K, Watanabe K. Gaze Cueing by Pareidolia Faces. -Percept. 2013 Dec;4(8):490-2.

47.52. Abubshait A, Wiese E. You Look Human, But Act Like a Machine: Agent Appearance and Behavior Modulate Different Aspects of Human-Robot Interaction. Front Psychol [Internet]. 2017 Aug 23;8. Available from:

http://journal.frontiersin.org/article/10.3389/fpsyg.2017.01393/full

48.53. Caruana N, de Lissa P, McArthur G. Beliefs about human agency influence the neural processing of gaze during joint attention. Soc Neurosci. 2016;12(2):194-206.

49.54. Wykowska A, Wiese E, Prosser A, Müller HJ. Beliefs about the Minds of Others Influence How We Process Sensory Information. Hamed SB, editor. PLoS ONE. 2014 Apr 8;9(4):e94339.

50.55. Wiese E, Wykowska A, Zwickel J, Müller HJ. I See What You Mean: How Attentional Selection Is Shaped by Ascribing Intentions to Others. Hamed SB, editor. PLoS ONE. 2012 Sep 26;7(9):e45391.

51.56. Epley N, Waytz A, Akalis S, Cacioppo JT. When We Need A Human: Motivational Determinants of Anthropomorphism. Soc Cogn. 2008;26(2):143-55.

52.57. Wiese E, Buzzell GA, Abubshait A, Beatty PJ. Seeing minds in others: Mind perception modulates low-level social-cognitive performance and relates to ventromedial prefrontal structures. Cogn Affect Behav Neurosci. 2018 Oct;18(5):837-56. 
53.58. Wiese E, Metta G, Wykowska A. Robots As Intentional Agents: Using Neuroscientific Methods to Make Robots Appear More Social. Front Psychol. 2017;8(October):1663.

54.59. Coffman BA, Clark VP, Parasuraman R. Battery powered thought: Enhancement of attention, learning, and memory in healthy adults using transcranial direct current stimulation. Neurolmage. 2014 Jan;85:895-908.

55.60. Jacobson L, Koslowsky M, Lavidor M. TDCS polarity effects in motor and cognitive domains: A meta-analytical review. Exp Brain Res. 2012;216(1):1-10.

56.61. Antal A, Nitsche MA, Paulus W. External modulation of visual perception in humans. Neuroreport. 2001;12(16):3553-5.

57.62. Cohen Kadosh R, Soskic S, Iuculano T, Kanai R, Walsh V. Modulating neuronal activity produces specific and long-lasting changes in numerical competence. Curr Biol. 2010;20(22):2016-20.

58.63. Nummenmaa L, Calder AJ. Neural mechanisms of social attention. Trends Cogn Sci. 2009 Mar;13(3):135-43.

59.64. Adams RB, Kleck RE. Effects of direct and averted gaze on the perception of facially communicated emotion. Emotion. 2005;5(1):3-11.

60.65. Blakemore SJ, Winston J, Frith U. Social cognitive neuroscience: where are we heading? Trends Cogn Sci. 2004;8(5):216-22.

61.66. Frischen A, Bayliss AP, Tipper SP. Gaze cueing of attention: Visual attentionn, Social Cognition, and Individual Differences. Psychol Bull. 2007;133(4):694-724.

62.67. Friesen CK, Kingstone A. The eyes have it! Reflexive orienting is triggered by nonpredictive gaze. Psychon Bull Rev. 1998;5(3):490-5.

63.68. Dalmaso M, Edwards SG, Bayliss AP. Re-encountering individuals who previously engaged in joint gaze modulates subsequent gaze cueing. J Exp Psychol Learn Mem Cogn. 2016;42(2):271-84.

64.69. Cazzato V, Liuzza MT, Caprara GV, Macaluso E, Aglioti SM. The attracting power of the gaze of politicians is modulated by the personality and ideological attitude of their voters: A functional magnetic resonance imaging study. Eur J Neurosci. 2015;42(8):253445.

65-70. Porciello G, Holmes BS, Liuzza MT, Crostella F, Aglioti SM, Bufalari I.

Interpersonal Multisensory Stimulation reduces the overwhelming distracting power of self-gaze: psychophysical evidence for 'engazement.' Sci Rep. 2014;4(6669):1-7.

66.71. Ciardo F, Marino BFM, Actis-Grosso R, Rossetti A, Ricciardelli P. Face age modulates gaze following in young adults. Sci Rep. 2014;4:4746.

67.72. Hungr CJ, Hunt AR. Physical self-similarity enhances the gaze-cueing effect. Q J Exp Psychol. 2012;65(7):1250-9.

68.73. Kawai N. Attentional shift by eye gaze requires joint attention: Eye gaze cues are unique to shift attention1: Social attention by the gaze cues. Jpn Psychol Res. 2011 Sep;53(3):292-301.

69.74. Bonifacci P, Ricciardelli P, Lugli L, Pellicano A. Emotional attention: effects of emotion and gaze direction on overt orienting of visual attention. Cogn Process. 2008 May;9(2):127-35. 
70.75. Fox MD, Snyder AZ, Vincent JL, Raichle ME. Intrinsic Fluctuations within Cortical Systems Account for Intertrial Variability in Human Behavior. Neuron. 2007;56(1):17184.

71.76. Tipples J. Fear and fearfulness potentiate automatic orienting to eye gaze. Cogn Emot. 2006;20(2):309-20.

72.77. Ristic J, Kingstone A. Taking control of reflexive social attention. Cognition. 2005 Jan;94(3):B55-65.

73.78. Caruana N, McArthur G, Woolgar A, Brock J. Simulating social interactions for the experimental investigation of joint attention. Neurosci Biobehav Rev. 2017 Mar;74:11525.

74.79. Wiese E, Wykowska A, Müller HJ. What We Observe Is Biased by What Other People Tell Us: Beliefs about the Reliability of Gaze Behavior Modulate Attentional Orienting to Gaze Cues. Hamed SB, editor. PLoS ONE. 2014 Apr 10;9(4):e94529.

75.80. Teufel C, Alexis DM, Todd H, Lawrance-Owen AJ, Clayton NS, Davis G. Social Cognition Modulates the Sensory Coding of Observed Gaze Direction. Curr Biol. 2009 Aug;19(15):1274-7.

76.81. Rosenthal-Von Der Pütten AM, Krämer NC. How design characteristics of robots determine evaluation and uncanny valley related responses. Comput Hum Behav. 2014;36:422-39.

77.82. Bartneck C. Robots in the theatre and the media. Des Semant Form Mov DeSForM2013. 2013;64-70.

78.83. Jack Al, Robbins P. The Phenomenal Stance Revisited. Rev Philos Psychol. 2012;3(3):383-403.

79.84. Krach S, Hegel F, Wrede B, Sagerer G, Binkofski F, Kircher T. Can machines think? Interaction and perspective taking with robots investigated via fMRI. PLoS ONE. 2008;3(7).

80.85. Takahashi H, Terada K, Morita T, Suzuki S, Haji T, Kozima H, et al. Different impressions of other agents obtained through social interaction uniquely modulate dorsal and ventral pathway activities in the social human brain. Cortex. 2014;58:289300.

81.86. Gobbini MI, Gentili C, Ricciardi E, Bellucci C, Salvini P, Laschi C, et al. Distinct Neural Systems Involved in Agency and Animacy Detection. J Cogn Neurosci. 2011;23(8):1911-20.

82.87. Carter EJ, Hodgins JK, Rakison DH. Exploring the neural correlates of goaldirected action and intention understanding. Neurolmage. 2011;54(2):1634-42.

83.88. Gobel MS, Tufft MRA, Richardson DC. Social Beliefs and Visual Attention: How the Social Relevance of a Cue Influences Spatial Orienting. Cogn Sci. 2017 May;42:16185.

89. Hackel LM, Looser CE, Van Bavel JJ. Group membership alters the threshold for mind perception: The role of social identity, collective identification, and intergroup threat. Journal of Experimental Social Psychology. 2014;52:15-23.

84.90. Cullen H, Kanai R, Bahrami B, Rees G. Individual differences in anthropomorphic attributions and human brain structure. Sc Cogn Affect Neurosci. 2013;9(9):1276-80. 
85.91. Apperly IA, Samson D, Chiavarino C, Humphreys GW. Frontal and TemporoParietal Lobe Contributions to Theory of Mind: Neuropsychological Evidence from a False-Belief Task with Reduced Language and Executive Demands. J Cogn Neurosci. 2004;16(10):1773-84.

86.92. Polanía R, Nitsche MA, Ruff CC. Studying and modifying brain function with noninvasive brain stimulation. Nat Neurosci. 2018 Feb;21(2):174-87.

87.93. Parkin BL, Ekhtiari H, Walsh VF. Non-invasive Human Brain Stimulation in Cognitive Neuroscience: A Primer. Neuron. 2015;87(5):932-45.

88.94. Bikson M, Datta A, Elwassif M. Establishing safety limits for transcranial direct current stimulation. Clin Neurophysiol. 2009 Jun;120(6):1033-4.

89.95. Oostenveld R, Praamstra P. The five percent electrode system for high-resolution EEG and ERP measurements. Clin Neurophysiol. 2001;7.

90.96. Blumberg EJ, Foroughi CK, Scheldrup MR, Peterson MS, Boehm-Davis DA, Parasuraman R. Reducing the Disruptive Effects of Interruptions With Noninvasive Brain Stimulation. Hum Factors. 2014;57(6):1051-62.

91.97. Falcone B, Coffman BA, Clark VP, Parasuraman R. Transcranial Direct Current Stimulation Augments Perceptual Sensitivity and 24-Hour Retention in a Complex Threat Detection Task. PLoS ONE. 2012;7(4):e34993.

92.98. Datta A, Truong D, Minhas P, Parra LC, Bikson M. Inter-Individual Variation during Transcranial Direct Current Stimulation and Normalization of Dose Using MRIDerived Computational Models. Front Psychiatry [Internet]. 2012 [cited 2018 Apr 6];3. Available from: http://journal.frontiersin.org/article/10.3389/fpsyt.2012.00091/abstract 93.99. Moliadze V, Antal A, Paulus W. Electrode-distance dependent after-effects of transcranial direct and random noise stimulation with extracephalic reference electrodes. Clin Neurophysiol. 2010 Dec;121(12):2165-71.

94.100. Bartneck C, Croft E, Kulic D. Measuring the anthropomorphism, animacy, likeability, perceived intelligence, and perceived safety of robots. Metr HRI Workshop Tech Rep. 2008;

95.101. Nomura T, Kanda T, Suzuki T. Experimental investigation into influence of negative attitudes toward robots on human-robot interaction. Al Soc. 2006;20(2):13850 .

96.102. Baron-Cohen S, Wheelwright S, Skinner R, Martin J, Clubley E. The AutismSpectrum Quotient (AQ): Evidence from Asperger Syndrome/High-Functioning Autism, Males and Females, Scientists and Mathematicians. J Autism Dev Disord. 2001;31(1):517.

97.103. Javadi AH, Cheng $\mathrm{P}$, Walsh V. Short duration transcranial direct current stimulation (tDCS) modulates verbal memory. Brain Stimulat. 2012 Oct;5(4):468-74. 98.104. Berryhill ME. Hits and misses: leveraging tDCS to advance cognitive research. Front Psychol [Internet]. 2014 [cited 2018 Aug 20];5. Available from: http://journal.frontiersin.org/article/10.3389/fpsyg.2014.00800/abstract

99.105. Boggio PS, Rocha RR, da Silva MT, Fregni F. Differential modulatory effects of transcranial direct current stimulation on a facial expression go-no-go task in males and females. Neurosci Lett. 2008 Dec;447(2-3):101-5. 
100-106. Ferrucci R, Mameli F, Guidi I, Mrakic-Sposta S, Vergari M, Marceglia S, et al. Transcranial direct current stimulation improves recognition memory in Alzheimer disease. Neurology. 2008 Aug 12;71(7):493-8.

101.107. Nitsche MA, Cohen LG, Wassermann EM, Priori A, Lang N, Antal A, et al. Transcranial direct current stimulation: State of the art 2008. Brain Stimulat. 2008;1(3):206-23.

102.108. Rani Das K, Imon, Rahmatullah. A Brief Review of Tests for Normality. Am J Theor Appl Stat. 2016;5(1):5.

103-109. Park YG. Comments on Statistical Issues in January 2013. Korean J Fam Med. 2013;34(1):64.

104.110. Schurz M, Radua J, Aichhorn M, Richlan F, Perner J. Fractionating theory of mind: A meta-analysis of functional brain imaging studies. Neurosci Biobehav Rev. 2014;42:934.

105.111. Chaminade T, Rosset D, Da Fonseca D, Nazarian B, Lutcher E, Cheng G, et al. How do we think machines think? An fMRI study of alleged competition with an artificial intelligence. Front Hum Neurosci [Internet]. 2012;6. Available from: http://journal.frontiersin.org/article/10.3389/fnhum.2012.00103/abstract

106.112. Caruana N, de Lissa P, McArthur G. The neural time course of evaluating selfinitiated joint attention bids. Brain Cogn. 2015 Aug;98:43-52.

107.113. Santiesteban I, Banissy MJ, Catmur C, Bird G. Enhancing Social Ability by Stimulating Right Temporoparietal Junction. Curr Biol. 2012 Dec;22(23):2274-7.

108.114. Santiesteban I, Banissy MJ, Catmur C, Bird G. Functional lateralization of temporoparietal junction - imitation inhibition, visual perspective-taking and theory of mind. Thut G, editor. Eur J Neurosci. 2015 Oct;42(8):2527-33.

109-115. Hogeveen J, Obhi SS, Banissy MJ, Santiesteban I, Press C, Catmur C, et al. Taskdependent and distinct roles of the temporoparietal junction and inferior frontal cortex in the control of imitation. Soc Cogn Affect Neurosci. 2015 Jul;10(7):1003-9.

110.116. Szczepanski SM, Knight RT. Insights into Human Behavior from Lesions to the Prefrontal Cortex. Neuron. 2014;83(5):1002-18.

111.117. Mitchell JP, Banaji MR, Macrae CN. General and specific contributions of the medial prefrontal cortex to knowledge about mental states. Neurolmage. 2005;28(4):757-62.

112.118. Fairhall SL, Anzellotti S, Ubaldi S, Caramazza A. Person- and place-selective neural substrates for entity-specific semantic access. Cereb Cortex. 2014;24(7):1687-96.

113.119. Contreras JM, Banaji MR, Mitchell JP. Dissociable neural correlates of stereotypes and other forms of semantic knowledge. Soc Cogn Affect Neurosci. 2012;7(7):764-70.

114.120. Simmons WK, Reddish M, Bellgowan PSF, Martin A. The selectivity and functional connectivity of the anterior temporal lobes. Cereb Cortex. 2010;20(4):813-25.

115.121. Ghosh VE, Moscovitch M, Melo Colella B, Gilboa A. Schema Representation in Patients with Ventromedial PFC Lesions. J Neurosci. 2014;34(36):12057-70.

116.122. Van Kesteren MTR, Ruiter DJ, Fern??ndez G, Henson RN. How schema and novelty augment memory formation. Trends Neurosci. 2012;35(4):211-9. 
117.123. Jenkins AC, Macrae CN, Mitchell JP. Repetition suppression of ventromedial prefrontal activity during judgments of self and others. Proc Natl Acad Sci U S A. 2008;105(11):4507-12.

118.124. Mitchell JP. Encoding-Specific Effects of Social Cognition on the Neural Correlates of Subsequent Memory. J Neurosci. 2004 May 26;24(21):4912-7.

119.125. Mitchell JP, Macrae CN, Banaji MR. Dissociable Medial Prefrontal Contributions to Judgments of Similar and Dissimilar Others. Neuron. 2006;50(4):655-63.

120.126. Wagner DD, Kelley WM, Heatherton TF. Individual differences in the spontaneous recruitment of brain regions supporting mental state understanding when viewing natural social scenes. Cereb Cortex. 2011;21(12):2788-96.

121.127. Kubit B, Jack Al. Rethinking the role of the rTPJ in attention and social cognition in light of the opposing domains hypothesis: findings from an ALE-based meta-analysis and resting-state functional connectivity. Front Hum Neurosci [Internet]. 2013;7.

Available from:

http://journal.frontiersin.org/article/10.3389/fnhum.2013.00323/abstract

122.128. Krall SC, Rottschy C, Oberwelland E, Bzdok D, Fox PT, Eickhoff SB, et al. The role of the right temporoparietal junction in attention and social interaction as revealed by ALE meta-analysis. Brain Struct Funct. 2015;220(2):587-604.

123.129. Wang Y, Quadflieg S. In our own image? Emotional and neural processing differences when observing human-human vs human-robot interactions. Soc Cogn Affect Neurosci. 2015;10(11):1515-24.

124.130. Mori M. The Uncanny Valley: The Original Essay by Masahiro Mori - IEEE Spectrum [Internet]. Energy. 1970. Available from: http://spectrum.ieee.org/automaton/robotics/humanoids/the-uncanny-valley

125.131. Balas B, Tonsager C. Face Animacy is Not All in the Eyes: Evidence from Contrast Chimeras. Perception. 2014 May;43(5):355-67.

126.132. Deska JC, Lloyd EP, Hugenberg K. Advancing Our Understanding of the Interface Between Perception and Intergroup Relations. Psychological Inquiry. 2016 Oct;27(4):286-9.

127.133. Gao T, McCarthy G, Scholl BJ. The Wolfpack Effect: Perception of Animacy Irresistibly Influences Interactive Behavior. Psychological Science. 2010 Dec;21(12):184553.

128.134. Schein C, Gray K. The Unifying Moral Dyad: Liberals and Conservatives Share the Same Harm-Based Moral Template. Personality and Social Psychology Bulletin. 2015 Aug;41(8):1147-63.

129.135. Looser CE, Wheatley T. The Tipping Point of Animacy: How, When, and Where We Perceive Life in a Face. Psychological Science. 2010 Dec 1;21(12):1854-62.

130.136. Wheatley T, Weinberg A, Looser C, Moran T, Hajcak G. Mind Perception: Real but Not Artificial Faces Sustain Neural Activity beyond the N170/VPP. Barnes S, editor. PLoS ONE. 2011 Mar 31;6(3):e17960.

131.137. Maurer D, Grand RL, Mondloch CJ. The many faces of configural processing. Trends in Cognitive Sciences. 2002 Jun;6(6):255-60. 
132.138. Zink CF, Kempf L, Hakimi S, Rainey CA, Stein JL, Meyer-Lindenberg A. Vasopressin modulates social recognition-related activity in the left temporoparietal junction in humans. Translational Psychiatry. 2011 Apr;1(4):e3-e3.

133.139. Perner J, Aichhorn M, Kronbichler M, Staffen W, Ladurner G. Thinking of mental and other representations: The roles of left and right temporo-parietal junction. Social Neuroscience. 2006 Sep;1(3-4):245-58.

134.140. Chaminade T, Hodgins J, Kawato M. Anthropomorphism influences perception of computer-animated characters' actions. Social Cognitive and Affective Neuroscience. 2007 Sep;2(3):206-16.

135.141. Gallagher $\mathrm{HL}$, Jack Al, Roepstorff A, Frith CD. Imaging the intentional stance in a competitive game. Neurolmage. 2002;16:814-21.

136.142. MacDorman KF, Entezari SO. Individual differences predict sensitivity to the uncanny valley. Interaction Studies. 2015;16(2):141-72. 